A Confidence Limit for the Empirical Mode Decomposition and Hilbert Spectral Analysis Author(s): Norden E. Huang, Man-Li C. Wu, Steven R. Long, Samuel S. P. Shen, Wendong Qu, Per Gloersen, Kuang L. Fan

Source: Proceedings: Mathematical, Physical and Engineering Sciences, Vol. 459, No. 2037 (Sep. 8, 2003), pp. 2317-2345

Published by: The Royal Society

Stable URL: http://www.jstor.org/stable/3560160

Accessed: 23/10/2009 05:26

Your use of the JSTOR archive indicates your acceptance of JSTOR's Terms and Conditions of Use, available at http://www.jstor.org/page/info/about/policies/terms.jsp. JSTOR's Terms and Conditions of Use provides, in part, that unless you have obtained prior permission, you may not download an entire issue of a journal or multiple copies of articles, and you may use content in the JSTOR archive only for your personal, non-commercial use.

Please contact the publisher regarding any further use of this work. Publisher contact information may be obtained at http://www.jstor.org/action/showPublisher?publisherCode=rsl.

Each copy of any part of a JSTOR transmission must contain the same copyright notice that appears on the screen or printed page of such transmission.

JSTOR is a not-for-profit service that helps scholars, researchers, and students discover, use, and build upon a wide range of content in a trusted digital archive. We use information technology and tools to increase productivity and facilitate new forms of scholarship. For more information about JSTOR, please contact support@jstor.org. 


\title{
A confidence limit for the empirical mode decomposition and Hilbert spectral analysis
}

\author{
By Norden E. Huang ${ }^{1}$, Man-Li C. W U ${ }^{2}$, Steven R. Long ${ }^{3}$, \\ Samuel S. P. Shen ${ }^{4}$, Wendong Q U ${ }^{5}$, Per Gloersen ${ }^{1}$ \\ AND KUANG L. FAN ${ }^{6}$ \\ ${ }^{1}$ Code 971, NASA Goddard Space Flight Center, \\ Greenbelt, $M D$ 20771, USA (norden.e.huang@nasa.gov) \\ ${ }^{2}$ Code 910, NASA Goddard Space Flight Center, Greenbelt, MD 20771, USA \\ ${ }^{3}$ Code 972, NASA GSFC/Wallops Flight Facility, Wallops Island, VA 23337, USA \\ ${ }^{4}$ Department of Mathematical Sciences, University of Alberta, \\ Edmonton T6G 2G1, Canada \\ ${ }^{5}$ Engineering Sciences, Mail Stop 104-44, California Institute \\ of Technology, Pasadena, CA 91125, USA \\ ${ }^{6}$ Institute of Oceanography, National Taiwan University, No. 1, Sec. 4, \\ Roosevelt Road, Taipei POB 23-13, Taiwan 106, Republic of China
}

Received 4 September 2001; accepted 14 January 2003; published online 23 July 2003

The confidence limit is a standard measure of the accuracy of the result in any statistical analysis. Most of the confidence limits are derived as follows. The data are first divided into subsections and then, under the ergodic assumption, the temporal mean is substituted for the ensemble mean. Next, the confidence limit is defined as a range of standard deviations from this mean. However, such a confidence limit is valid only for linear and stationary processes. Furthermore, in order for the ergodic assumption to be valid, the subsections have to be statistically independent. For non-stationary and nonlinear processes, such an analysis is no longer valid. The confidence limit of the method here termed EMD/HSA (for empirical mode decomposition/Hilbert spectral analysis) is introduced by using various adjustable stopping criteria in the sifting processes of the EMD step to generate a sample set of intrinsic mode functions (IMFs). The EMD technique acts as a pre-processor for HSA on the original data, producing a set of components (IMFs) from the original data that equal the original data when added back together. Each IMF represents a scale in the data, from smallest to largest. The ensemble mean and standard deviation of the IMF sample sets obtained with different stopping criteria are calculated, and these form a simple random sample set. The confidence limit for EMD/HSA is then defined as a range of standard deviations from the ensemble mean. Without evoking the ergodic assumption, subdivision of the data stream into short sections is unnecessary; hence, the results and the confidence limit retain the full-frequency resolution of the full dataset. This new confidence limit can be applied to the analysis of nonlinear and non-stationary processes by these new techniques. Data from length-of-day measurements and a particularly violent recent earthquake are used to demonstrate how the confidence limit is obtained and applied. By providing a confidence limit for this new approach, a stable range of stopping criteria for the decomposition or sifting phase 
(EMD) has been established, making the results of the final processing with HSA, and the entire EMD/HSA method, more definitive.

Keywords: empirical mode decomposition; EMD/HSA; Hilbert spectral analysis; Hilbert--Huang transform (HHT); nonlinear data analysis; non-steady data analysis

\section{Introduction}

The two-step method of empirical mode decomposition (EMD) and Hilbert spectral analysis (HSA) introduced by Huang et al. (1998a, 1999a, hereafter referred to as H98 and H99) has proved to be a powerful procedure for analysing non-stationary and nonlinear data. During the years since its introduction, many applications have been found (Huang 2001; Huang et al. 1998b, 1999b, 2000, 2001; Gloersen \& Huang 1999; Wu et al. 1999; Loh et al. 2001; Hu et al. 2002) that include analysing acoustic, biological, ocean, earthquake, climate and mechanical vibration data. As versatile as it has proved to be, the method still needs further clarifications and improvements. An aspect in need of development and clarification is the definition and analysis of a confidence limit for the resulting intrinsic mode functions (IMFs) and the Hilbert spectrum.

The confidence limit is a standard measure for results from statistical analysis. Ideally, it should be derived from an ensemble of observations and computed using the ensemble mean and standard deviation from this mean. Assuming the error is normally distributed, the confidence limit is usually defined as a range of values near this mean: one standard deviation is equivalent to $68 \%$, and two standard deviations are equivalent to a $95 \%$ confidence limit. For practical reasons, however, only a few of the many statistical analysis studies follow this rule. Most of the studies used a confidence limit computed from one set of observations only, instead of an ensemble; the mean and the standard deviation are actually computed by invoking the ergodicity, assuming that the data are linear and stationary, and the data can be subdivided into statistically independent subsections. The temporal mean from these subsections is then used to approximate the ensemble mean.

The ergodic rule seems straightforward, but there are certain difficulties involved that are consistently overlooked. The first difficulty is that there may not be enough data values to allow subdivision of the dataset into enough subsets so that each will have enough data to represent the process and allow a realistic mean to be computed within each subset. And the more troublesome hurdle is that the data may not be stationary or from a linear process. Once the stationary assumption is abandoned, the ergodicity can no longer be assumed to compute the mean and standard deviation from different subsections of the same given dataset; a true ensemble mean is required. Unfortunately, in the real world, only one realization is available, which gives only one dataset: no one can demand and achieve a repeat of a natural process even once, much less so enough for an ensemble average.

The most serious objection is that natural processes are not only non-stationary but also nonlinear, which involves sensitivity to the initial conditions and reactions to feedback. Thus, most of the underlying physical processes make the ergodic assumption inapplicable, if not irrelevant. Therefore, a statistical measure of the result cannot simply be established by resorting to spatial and temporal averaging; yet finding an alternative is a grand challenge. 
With the new two-step method of EMD/HSA, there are many possible selections of free parameters, such as the maximum number of sifting times and the stopping criteria for extracting each intrinsic mode function (IMF). Each of the parameters can be selected independently of the others, and there are few, if any, solid guidelines to follow in the selection of these parameters. The obvious (yet critical) question is which set of the many possible choices of sifting parameters gives a meaningful result. This also leads to further questions: how can the goodness or the reliability of any result be measured? Can these different free parameters be used in some way to generate a random sample set of results, and even define a confidence limit on the sample?

The intent of this paper is to suggest a method to establish just such a confidence limit for this new approach as a statistical measure of the fidelity of the result without invoking the ergodic assumption. The EMD (the first step of the EMD/HSA method) can generate infinitely many IMF sets from a given set of data by changing the starting conditions for the first-step processing. This capability will be used to generate a simple random sample set. To obtain the sample, the sifting parameters will be judiciously selected so that the members of the sample set still retain physically meaningful characteristics. By this means, a simple random sample set is obtained from a single data realization. A mean of the sample set is then found, which will also still be a function of time. The non-stationary characteristics of the data are thus fully preserved in the results.

As the method for establishing a confidence limit depends on variations of the sifting parameters, the sifting procedures are first discussed in some detail, including the stopping criteria, the maximum number of siftings, intermittence, sifting methods, the nomenclature needed for the confidence limit, and how the results from the first step of the processing are carried into the second step of HSA. These procedures will then be applied to the length-of-day (LOD) dataset with an emphasis on the selection of the various sifting parameters that will create the simple random sample set of sifted results, which will lead to the establishment of the proposed confidence limit. The results are then used to find the mean of the simple random sample set and the deviation of individual cases to establish an optimal range of sifting parameters, sampling strategy and optimal stopping of the first-step processing for IMFs. To demonstrate the techniques for more general data without fixed cycles, data from an exceptionally powerful earthquake are used.

By establishing a confidence limit for the EMD/HSA approach as well as outlining procedures to optimize this two-step process, the method is made more robust and useful for the many applications already underway in the study of nonlinear and unsteady processes.

\section{The sifting process (EMD) and the selection of sifting parameters}

There are infinitely many ways to decompose a given dataset. The EMD is one method that can generate infinitely many and different IMF sets through different sifting parameters. As discussed in Huang et al. (1996), H98 and H99, the EMD method is the procedure needed to generate the adaptive IMF basis. An IMF is defined as any function satisfying the following conditions:

(i) in the whole dataset, the number of extrema and the number of zero crossings must either equal or differ by at most one; 
(ii) at any point, the mean value of the envelope defined by the local maxima and the envelope defined by the local minima is zero.

Thus, an IMF represents a simple oscillatory mode as a counterpart to the simple harmonic function of the Fourier series method, but it is much more general, for it is a function with both amplitude and frequency modulations. To extract the IMF from a given dataset, the sifting process is implemented as follows. Identify all the local extrema, and then connect all of the local maxima by a cubic spline line as the upper envelope. Repeat the procedure for the local minima to produce the lower envelope. The upper and lower envelopes should cover all the data between them. Their mean is designated $m_{1}$, and the difference between the data and $m_{1}$ is $h_{1}$, i.e.

$$
X(t)-m_{1}=h_{1} .
$$

The detailed procedure for this is illustrated in more detail in H98. Ideally, $h_{1}$ should be an IMF, for the construction of $h_{1}$ described above should have forced the result to satisfy all the definitions of an IMF by construction. Yet, after the first round of sifting, a local hump near an inflection point (which is not an extremum in the original rectangular coordinates) may become a local maximum and minimum when the coordinate system is changed to the curvilinear one with $m_{1}$ as the zero reference. New extrema generated in this way are necessary to recover the intrinsic modes lost in the initial examination. Therefore, the function $h_{1}$ can only be designated as a proto-mode function (PMF). The PMF has to be processed further to yield the true IMF through repetitive processing. We term these repeated processing steps as 'sifting', due to the order in which the components emerge: the smallest scales first, followed by larger scales that increase with each 'sifting'. The challenge, therefore, is to determine a rigorous stopping criterion to produce a physically meaningful IMF.

The sifting operations implemented in the first-step processing of the EMD/HSA technique contain the following parameters: the stopping criteria for producing an IMF component, and the maximum number of sifting steps to be allowed. Furthermore, a decision is needed on whether or not to invoke the intermittence test. Finally, the choice of whether to use local extrema or curvature extrema as the basis to define the envelopes must be made. These choices are explained in detail in the following.

\section{(a) The stopping criterion for producing an IMF}

The 'stopping criterion' actually determine the number of sifting steps to produce an IMF; it is of critical importance in the successful implementation of the EMD processing. As described above, the PMF needs to be further refined. Therefore, the sifting process has to be repeated as many times as necessary, to eliminate all the riding waves. To start the subsequent sifting process steps, $h_{1}$ is treated as the data. Then

$$
h_{1}-m_{11}=h_{11},
$$

where $m_{11}$ is the mean of the upper and lower envelopes of $h_{1}$. This process can be repeated up to $k$ times; $h_{1 k}$ is then given by

$$
h_{1(k-1)}-m_{1 k}=h_{1 k} .
$$

After each processing step, checking must be done on whether the number of zero crossings equals the number of extrema. 


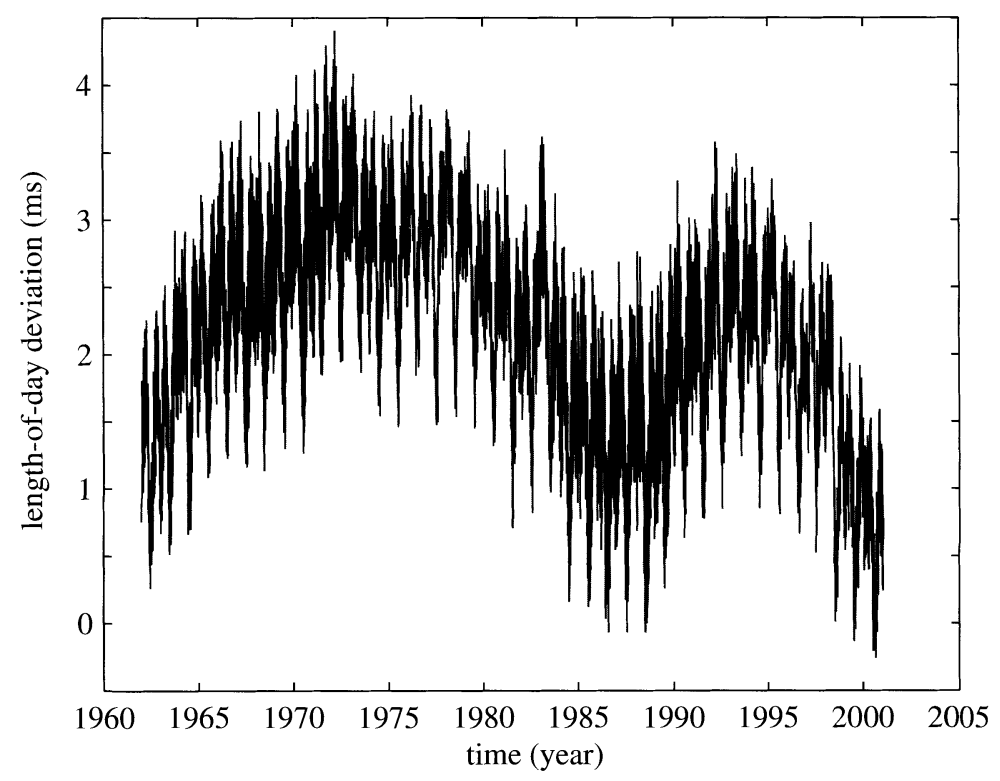

Figure 1. The daily LOD data, extracted from the comb2000_daily.eop series constructed by Gross (2001).

The sifting process actually serves two purposes: to eliminate riding waves and to make the wave profiles more symmetric with respect to zero. If eliminating riding waves and forcing the mean to be zero are the only requirements, sifting should be done as many times as needed to satisfy them. Unfortunately, in the process of achieving these goals, the sifting process also produces a side effect: to make the neighbouring wave have more even amplitudes. While the former two effects are essential for computing instantaneous frequency through the Hilbert transform, the side effect actually decreases the range of variations in the IMF by smoothing the amplitudes. As simple as the procedure described here really is, the implementation is far more subtle: the effects of sifting must be balanced with its side effects. Therefore, two conflicting requirements must be addressed. To eliminate the riding waves and to force a local zero, sifting as many times as possible is needed. On the other hand, too many sifting steps will reduce the IMF to be a constant-amplitude frequency-modulated function, which would obliterate the intrinsic amplitude variations and render the results physically less meaningful. To preserve the natural amplitude variations of the oscillations, sifting must be limited to as few steps as are mathematically permissible; the choice must be selected judiciously. What exactly the stopping criterion should be is a difficult decision. Two possibilities have been proposed. The first one, used in H98, is the Cauchy-type convergence criterion, where the sifting is carried to the point when the difference between the successive sifted results is smaller than a preset limit. The major flaw of that approach is that it is unrelated to the definition of the IMF; it only requires the successive PMFs to be approximately equal. Thus, something better was needed.

The second criterion was proposed by H99, where the sifting is stopped when the number of zero crossings and extrema is the same number for $S$ successive sifting steps. The first task in this approach is to determine a number $S$. Thus, the stopping 


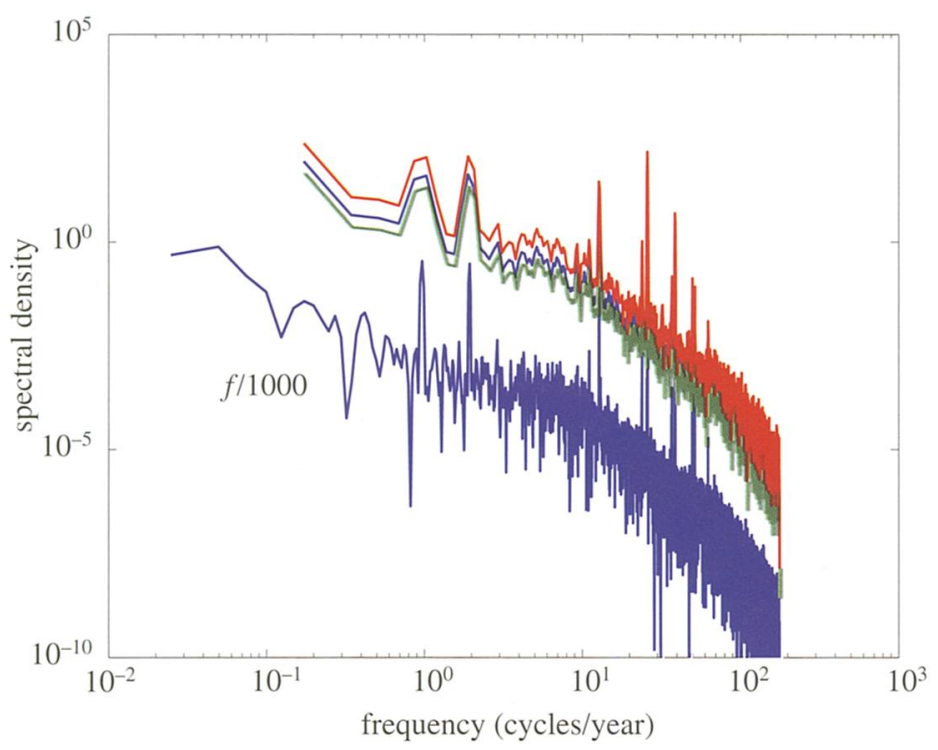

Figure 2. The Fourier spectrum of the LOD data (with its magnitude shifted by three decades for easier viewing) and the subdivided data to produce the confidence limit.

critcrion provides a soft boundary for the sifting procedure; slightly different results from a given set of data can be obtained by adopting different $S$ values. It is this variation of $S$ that will be used to generate a simple random sample set of IMFs with the following procedure: first select different $S$ values and get different resulting IMF sets. For example, if $S_{1}$ is selected as the stopping criterion (that is, the sifting stops when $S_{1}$ successive sifting steps produce the same number of extrema and zero crossings; for example, let the sifting stop at the $k$ th sifting), then

$$
c_{1}=h_{1 k}
$$

is designated as the first IMF component from the data. Overall, $c_{1}$ should contain the finest-scale or the shortest-period component of the signal corresponding to this stopping condition.

In the next trial, another number for the stopping criterion is selected as $S_{2}$, resulting in sifting to the $m$ th step to satisfy the definitions of an IMF, and, of course, $h_{1 m}$ may not necessarily equal $h_{1 k}$. Many $S$ values are thus selected and produce as many sets of IMFs. They all represent the truth approximately, while some might be closer to the truth than others; there is no objective way to determine which one of the IMF sets is the best. The orthogonal index (OI) can be used as a criterion to reject those IMF sets that are grossly non-orthogonal. Within the acceptable range of the OI, each set must be treated equally. In this way, a simple random sample set can be generated from the equally valid IMF sets. From such a sample set, the mean and standard deviation can be computed as a statistical measure of the sifting results.

In general, the higher the $S$ number selected, the higher the total number of sifting steps required. As over-sifting can have a detrimental effect on the results, do not select too high a number for the stopping criterion. Typically, a value of $3 \lesssim S \lesssim 5$ has proved successful as the default stopping criterion. 

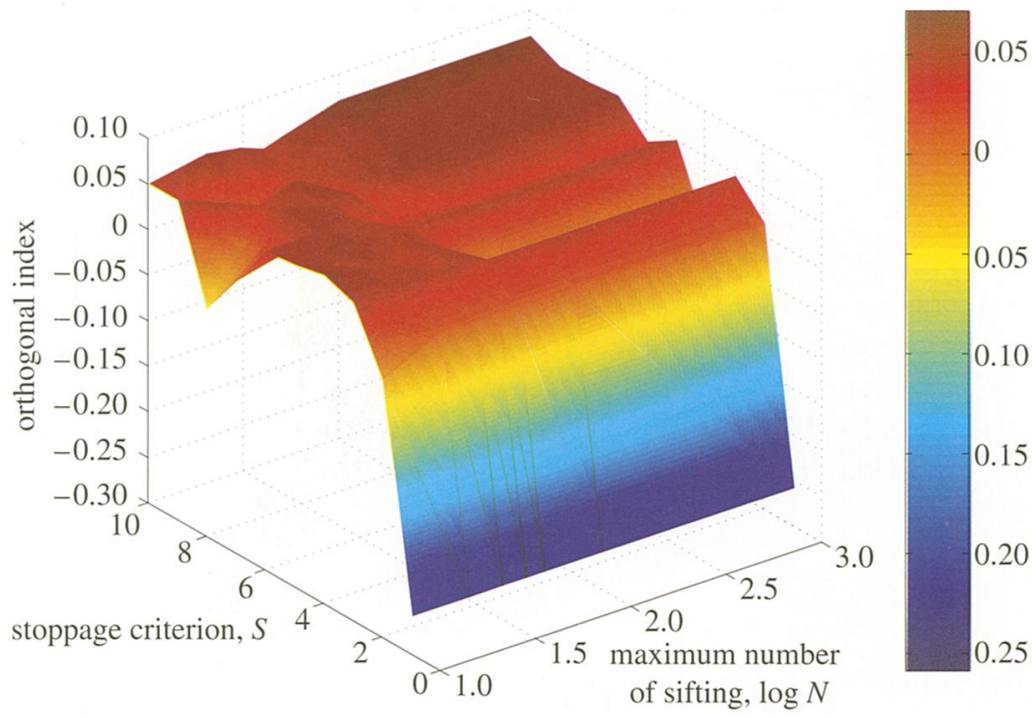

Figure 3. The 3D view of the OI variations as a function of the maximum sifting times and the stoppage number.

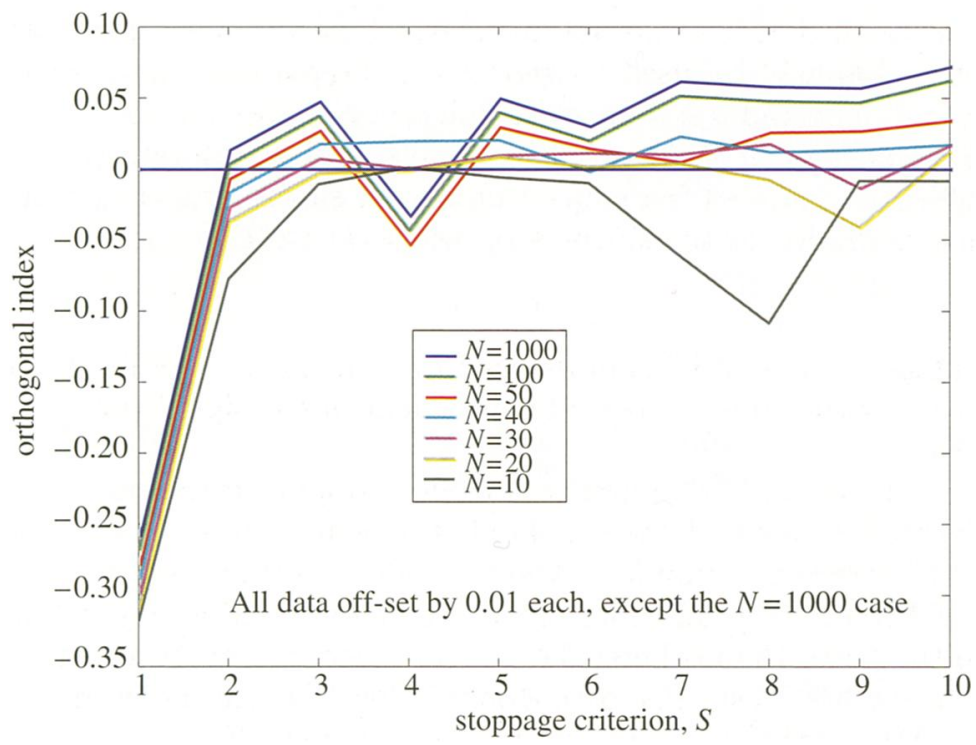

Figure 4. Multiple cuts of the 3D view given in figure 3 of OIs along fixed maximum sifting times, with each curve shifted 0.01 downward for easier viewing.

Once $c_{1}$ is obtained, it can be separated from the rest of the data by using

$$
X(t)-c_{1}=r_{1} .
$$

Since the residue, $r_{1}$, still contains longer-period components, it is treated as the original complete input data and subjected to the same sifting process as described 
above. This procedure can be repeated for all the subsequent $r_{j}$ values, and the result is

$$
\begin{gathered}
r_{1}-c_{2}=r_{2}, \\
\vdots \\
r_{n-1}-c_{n}=r_{n} .
\end{gathered}
$$

The sifting process should continue until the residue, $r_{n}$, becomes a constant value, a monotonic function or a function with only one extremum from which no more IMFs can be extracted. Even for data with a zero mean, the final residue can still be different from zero. If the data have a trend, the final residue will be that trend. By summing equations (2.5) and (2.6), it follows that

$$
X(t)=\sum_{j=1}^{n} c_{j}+r_{n} .
$$

The sifting processes are finished and a set of IMFs has been produced. Next, the stopping criterion is changed to $S_{3}$, which produces another set of IMFs. This can be repeated as many times as needed, and thereby can produce a simple random sample set from the IMFs.

\section{(b) The maximum number of siftings}

Selecting a maximum number of siftings, $M$, serves two purposes: to guarantee that over-sifting does not occur, and to prevent the sifting procedures from locking in a never-ending loop. The former concern can be eliminated by selecting a low enough $S$; the latter concern has never been a problem. The selection of this number will be shown not to be as critical as the stopping criterion. However, this number should be set large enough to guarantee that the selected $S$ is fully implemented.

\section{(c) Intermittence}

The intermittence criterion has been discussed in great detail in H99. Due to its importance, it will be reviewed briefly here. Intermittence is a signature of turbulence in the fluid motion (see, for example, Frisch 1995); therefore, it is natural to encounter data with intermittent variations. As discussed in H99, the existence of intermittence can produce mode mixing, a phenomenon caused by having different time-scales (or spatial scales) mixed in a single IMF component, which will introduce additional, but fictitious, variations in the resulting IMFs and, hence, in the instantaneous frequency values. The intermittence criterion requires the selection of a number such as $n_{1}$. This number represents the number of data points that correspond with a chosen data limit: only the waves shorter than this limit are to be included in a given IMF. If the distance between the successive extrema is larger than $n_{1}$, the mean is used to replace that data point. This criterion requires that only when the distance between the extrema is less than $n_{1}$ will the upper and lower envelopes and the mean be available to extract the IMFs. This criterion is difficult to set a priori, unless there is a strong theoretical basis to establish a distinct scale length. Even in such cases, a priori judgment could still eliminate interesting physical variations 
(a)

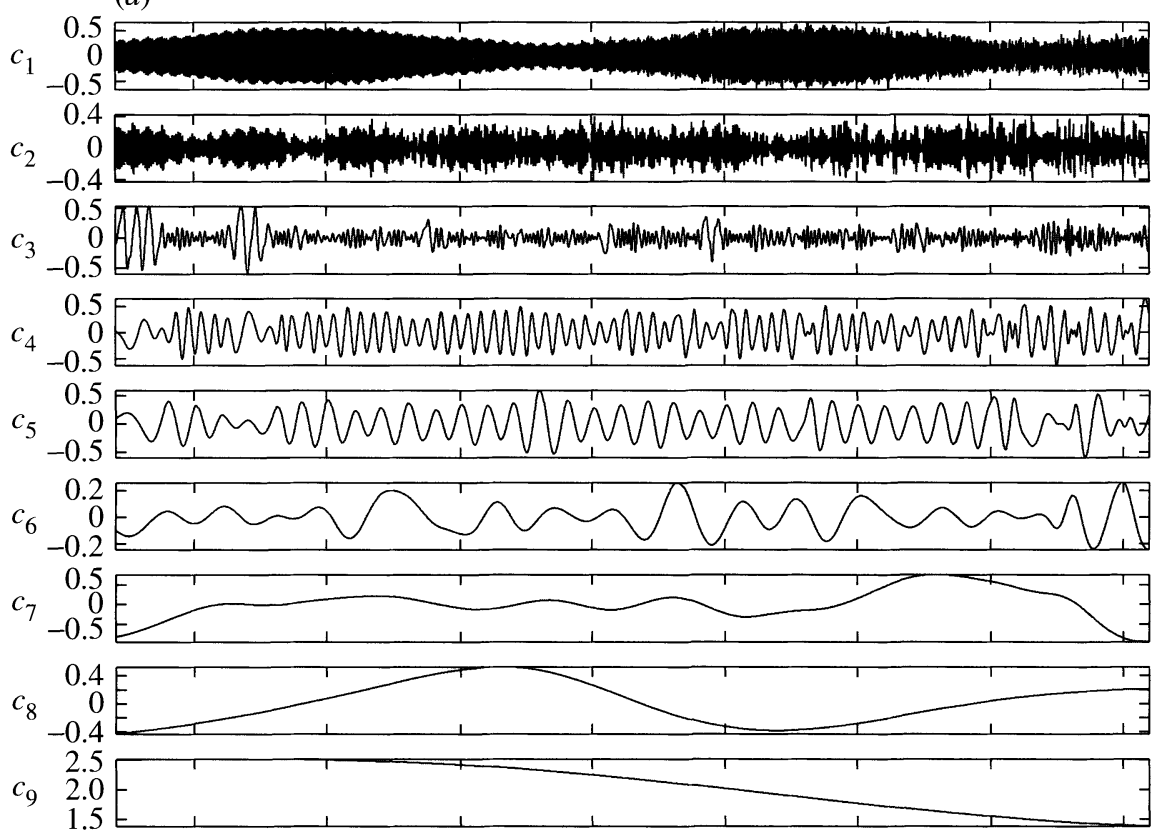

(b)

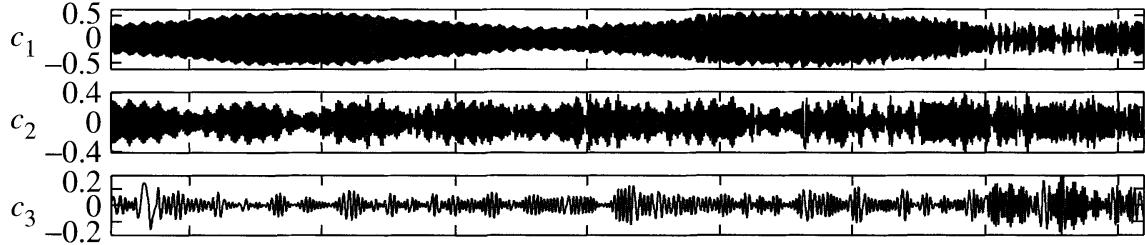

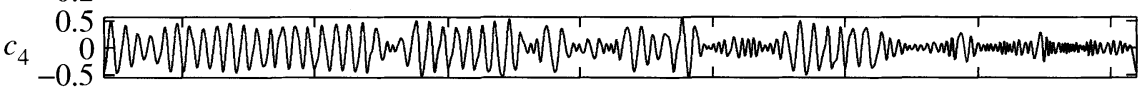

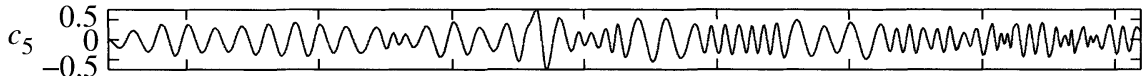

$c_{6}{ }_{-0.5}^{0.5}$

$c_{7}{ }_{-0.2}^{0.2}$

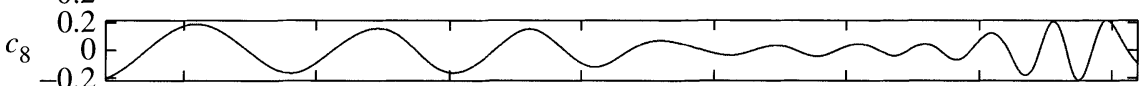

$c_{9}$

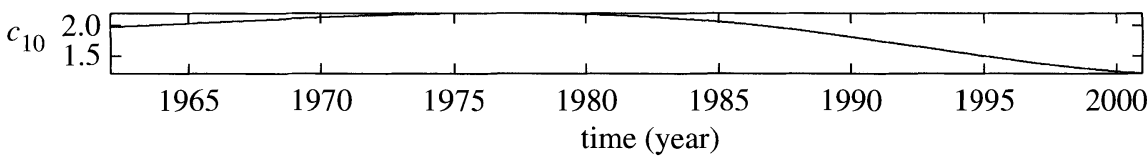

Figure 5. The IMF components from extrema-based sifting without intermittency testing. (a) An individual case, $\mathrm{CE}(100,2)$. (b) An individual case, $\mathrm{CE}(100,10)$. 

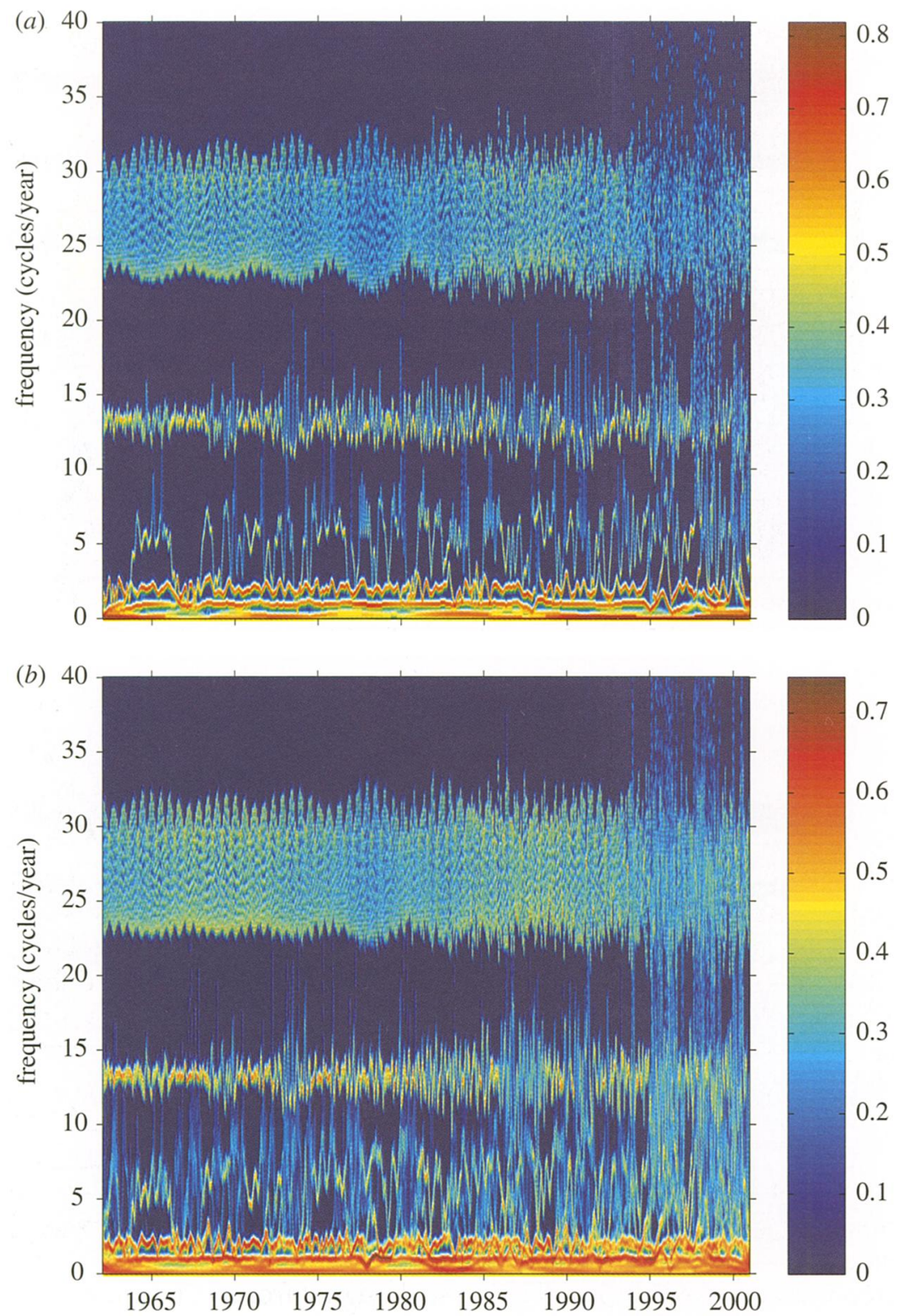

Figure 6 . The Hilbert spectra constructed from the CE series of siftings with $7 \times 7$ smoothing: (a) the individual case for the Hilbert spectrum based on $\mathrm{CE}(100,2) ;(b)$ the mean Hilbert spectra of 11 different siftings based on the CE series.

in the data. As in the example shown in $\S 4$, when a special scale exists but is not separated from the rest of the data properly, there is the risk of a loss of focus on the underlying process. In that example case, the process with the special scale would be fragmented with many added amplitude variations distributed into several different IMF components. 


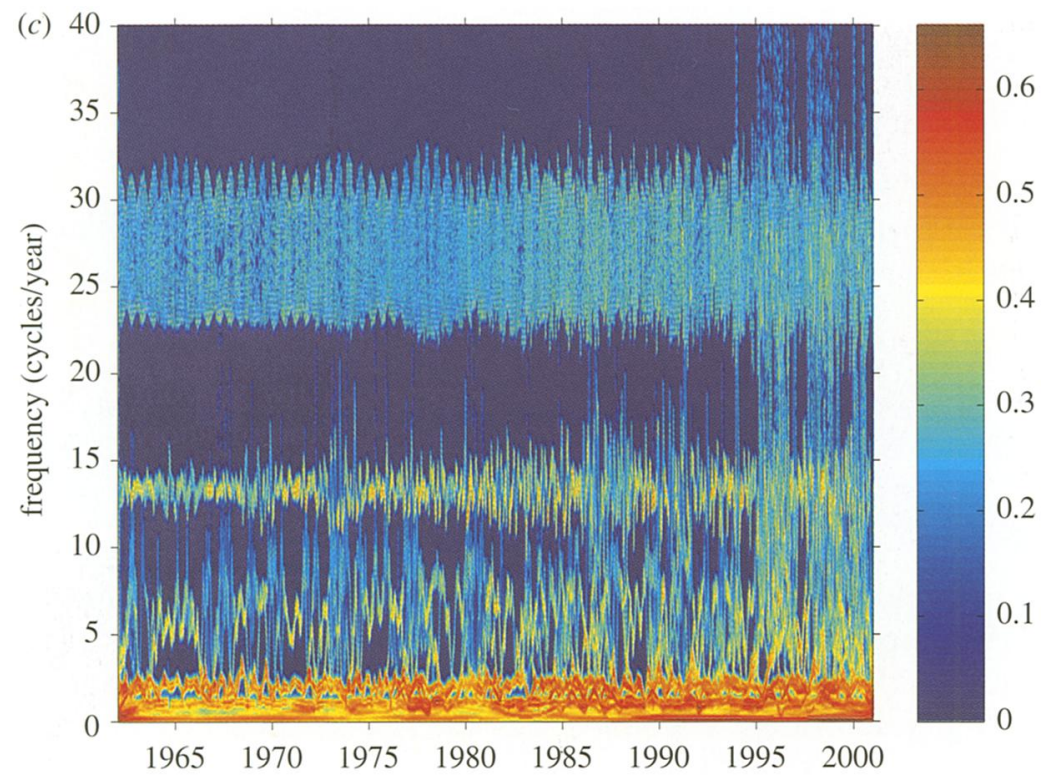

Figure 6. (Cont.) (c) The standard deviation of the Hilbert spectra from 11 different siftings based on the CE series.

To avoid the above difficulties, we start by processing all data without invoking the intermittence criteria. If serious mode mixing is detected, we invoke the intermittence test by setting a criterion to ignore waves (or other data scales) with a period longer than a preset length. By doing this for the successive IMF components, all the oscillatory signals of a similar length can be included in a single IMF. This procedure is similar to filtering in temporal space; it rejects signals of drastically different lengthscales. It is an effective step to extract a signal of a given length-scale, as will be illustrated later in the example of the LOD data.

\section{(d) Curvature- or extrema-based sifting}

As discussed in H99, the extrema points can be used as a basis to establish the upper and lower envelopes for sifting. But the extrema basis will miss the gentle humps characterized as hidden scales. By computing the curvature, it is possible to get one step further in extracting the fine-scale signal from a given data. By the same token, higher and higher derivatives can be taken, and thus we get finer and finer scale signals. Where should the processing stop? H99 proposed to stop at the curvature with the justification that if the data represent the displacement, the curvature is equivalent to the acceleration. Within the framework of Newtonian mechanics, derivatives higher than this will cease to have any physical meaning. Of course, not all data represent displacement; therefore, higher derivatives may still be meaningful in some cases. If that is true, the situations must be examined individually. But a more practical limitation still needs to be heeded: all data contain noise, and differentiation is a noise amplifier, if not a noise-generating mechanism. Therefore, the result from high-order differentiation would be unavoidably contaminated by noise. 
It must be noted that, by choosing a curvature-based sifting, the meaning of the data is totally different from that for the extrema-based sifting. For example, consider the function

$$
x(t)=\cos (\omega t+\varepsilon \sin 2 \omega t)
$$

for small $\varepsilon$; this is an IMF already based on extrema. According to the classical wave analogy, this signal should be just a frequency-modulated oscillation with the time-varying frequency given by

$$
\Omega=\omega(1+2 \varepsilon \cos 2 \omega t) .
$$

If curvature-based sifting is selected, the form will be very different, as the second derivative of the data will become

$$
x^{\prime \prime}(t)=-(\omega+2 \varepsilon \omega \cos 2 \omega t)^{2} \cos (\omega t+2 \varepsilon \sin 2 \omega t)+4 \varepsilon \omega^{2} \sin 2 \omega t \sin (\omega t+2 \varepsilon \sin 2 \omega t) .
$$

In this form, the curvature-based sifting will produce more than one time-scale in different IMF components. For this case, this result is not necessarily better. In fact, it actually masks the clear representation of the frequency modulation given in the expression of equation (2.9). Therefore, the selection of the envelope base should be made with great care. Experience recommends using the extrema-based sifting as a first trial. The curvature or higher-order-derivative-based siftings should only be invoked for special cases when the result suggests too many hidden scales.

\section{(e) Nomenclature of the decomposition and confidence limit}

With all the above possibilities of parameter selections, there are almost infinitely many possible combinations, giving an infinite number of possible decompositions from a single set of data. To identify them, the choice of all the parameters involved in the sifting process should be specifically identified. As a way to designate the results, the following nomenclature is proposed: $\mathrm{CE}(M, S)$, for extrema-based sifting (Components from Extrema, CE), with $M$ as the maximum number of sifting steps allowed, and $S$ as the stopping criterion; $\mathrm{CC}(M, S)$, for the corresponding curvature-based sifting; $\mathrm{CEI}\left(M, N ; n_{1}, n_{2}, \ldots\right)$, for the extrema-based sifting with intermittency testing, while the wavelength for the first, second, and following $(. .$. components are set at $n_{1}, n_{2}, \ldots$; and $\mathrm{CCI}\left(M, N ; n_{1}, n_{2}, \ldots\right)$, for the corresponding curvature-based sifting with intermittence. When the same intermittence criterion, say $n_{2}$, is used repeatedly for $k$ times, this will be denoted as a repeated application of $n_{2}$ by $n_{2}^{k}$ as a shorthand notation.

The various IMF sets from different sifting criteria are equally valid representations of the data, provided their OIs are within a preset limit, 0.1 say. Collectively, they form an ensemble of samples. Therefore, a simple random sample set can be obtained, along with the mean and standard deviations, all without invoking the ergodic assumption. Consequently, the special conditions required for ergodicity would be irrelevant here. But we do have to make sure that the sampling strategy is a fair one.

There is only one difficulty in carrying out the averaging: the IMF sets may not all consist of the same number of components. Under such conditions, it is possible to implement the averaging to the Hilbert spectra, which can have the same number of bins in frequency and temporal space pre-assigned. For simplicity, plus and minus one 
standard deviation for the $68 \%$ confidence level has been adopted (or plus and minus two standard deviations for the $95 \%$ confidence level) in the subsequent illustrations. Having thus established the confidence interval of the sifting process, the optimal number for $S$ will be delayed until after the examples.

\section{(f) Hilbert spectrum}

Once the decomposition is complete, the data are represented in terms of the adaptive basis function as in equation (2.7). In this form, the data are still in temporal space. To transform this temporal-space data to time-frequency space, a Hilbert transform is performed on each IMF component, and the amplitude and instantaneous frequency for every IMF at every time-step is computed. This result can be projected on the time-frequency-energy space, with energy defined as the amplitude squared. The resulting time-frequency-energy representation of the data is defined as the Hilbert spectrum. In performing the Hilbert transform, the last residual is normally omitted, as it is not an IMF. Under special conditions, however, it could be included, if the physical conditions require it.

The Hilbert spectrum presents a different view of the data. It is very useful in regrouping the decomposed data in the time-frequency space. For example, when a dataset is decomposed into different IMF sets with different total components and severe mode mixing, the Hilbert spectrum will still assign the energy to the correct time-frequency location, albeit with some alias at the mode transition locations. A time integration of the Hilbert spectrum is a reduced frequency-energy representation and is defined as the marginal spectrum. This is only the projection of the true three-dimensional (3D) full representation; therefore, it should not be used routinely. However, it does provide a convenient form for comparison with the conventional Fourier spectrum representation.

\section{An example: analysis of the length-of-day data}

Having established the sifting procedures, the EMD/HSA two-step method will now be applied to the LOD data as an example to illustrate the procedures. This two-step method is also referred to as the Hilbert-Huang transform (HHT). The daily LOD dataset was produced by Gross (2001), covering the period from 20 January 1962 to 6 January 2001, for a total of 14232 days. The raw data are from independent Earthorientation measurements taken by the space-geodetic techniques that include lunar and satellite laser ranging, very-long-baseline interferometry, the Global Positioning System and optical astrometric measurements. These data are combined using the Kalman filter. The solid Earth and oceanic tides were first removed and later reintroduced by using the models of Yoder et al. (1981) for the solid Earth tides, and Kantha et al. (1998) for the oceanic tides without the semidiurnal and diurnal components. A detailed discussion of the LOD data can be found in Gross $(1996,2000$, 2001) and Gross et al. (1998). These data are available at ftp://euler.jpl.nasa.gov/ keof/combinations/2000. The data, shown in figure 1 and used here to illustrate the EMD/HSA method, are taken from the comb200_daily.eop series from the above Web site.

First, these data will be examined using Fourier spectral analysis. The result is given in figure 2, where the Fourier spectra from the total dataset (with its magnitude lower by three decades), the mean spectrum and confidence limit from seven 


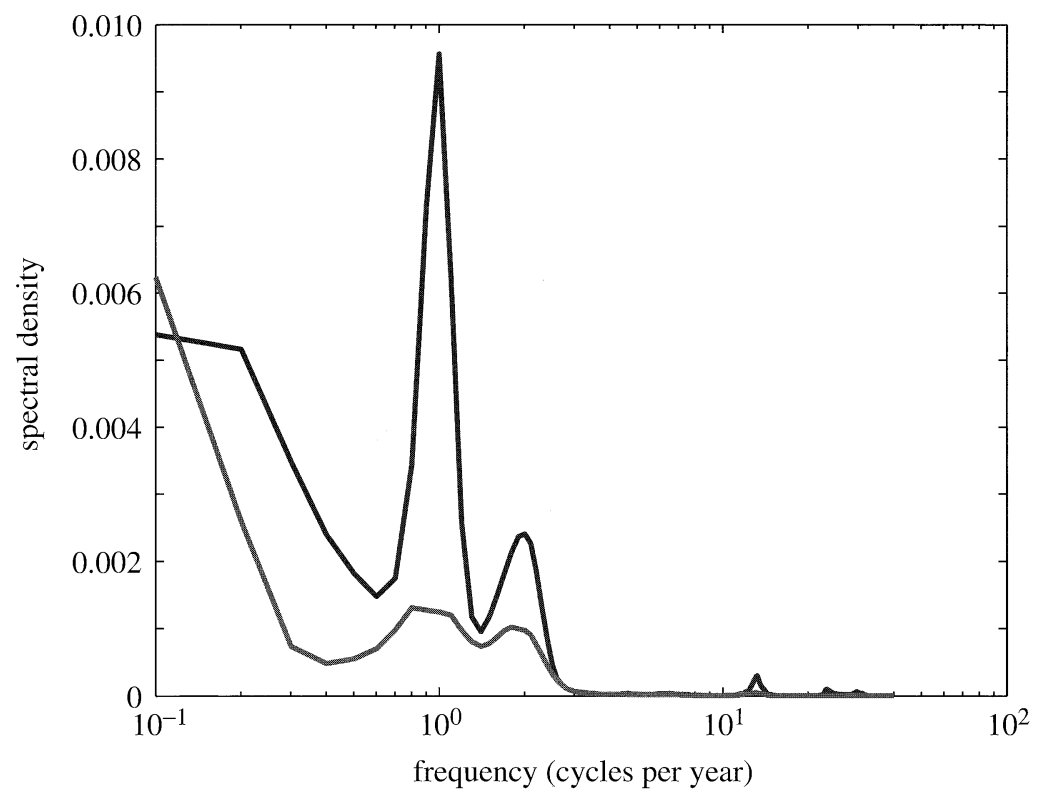

Figure 7. The marginal spectra for the CE series from the mean Hilbert spectrum and the time frequency representation of the standard deviation of the Hilbert spectra.

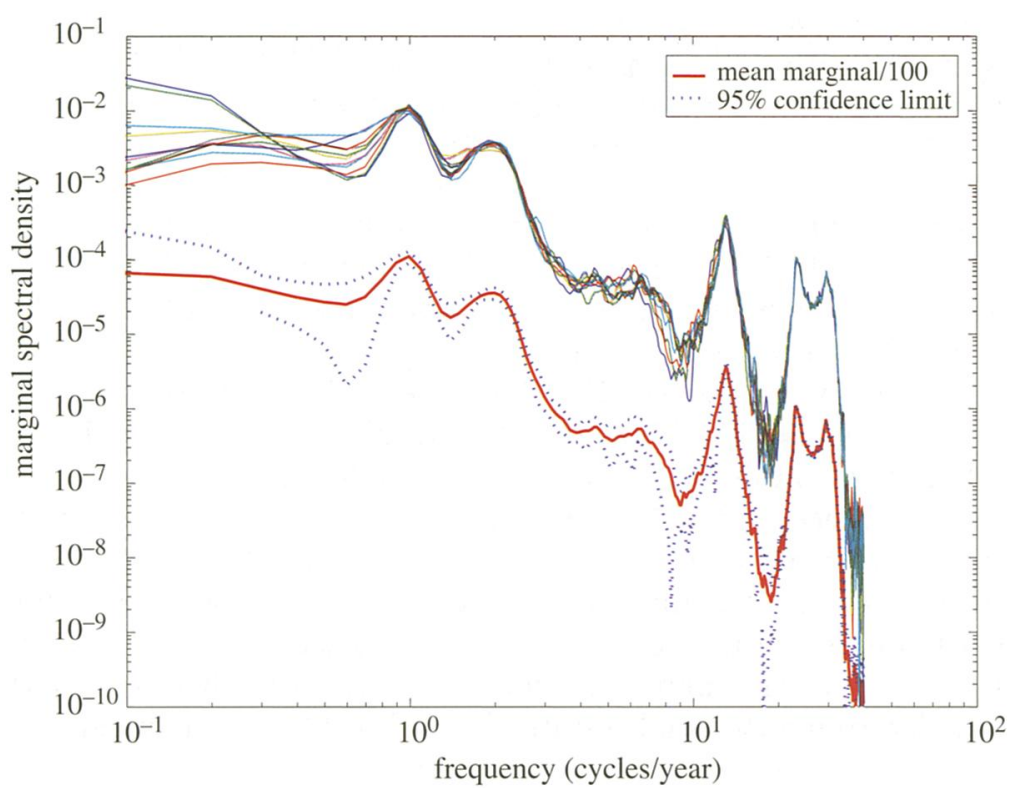

Figure 8. All 11 marginal Hilbert spectra for the CE series, and their mean (shifted down two decades) with the two-standard-deviation confidence limit.

subsections are given. The smoothing processes cause the magnitudes of the spectral peaks to decrease and the frequency band to widen. These changes are all governed by the uncertainty principle. By taking the subsections, however, the information in 


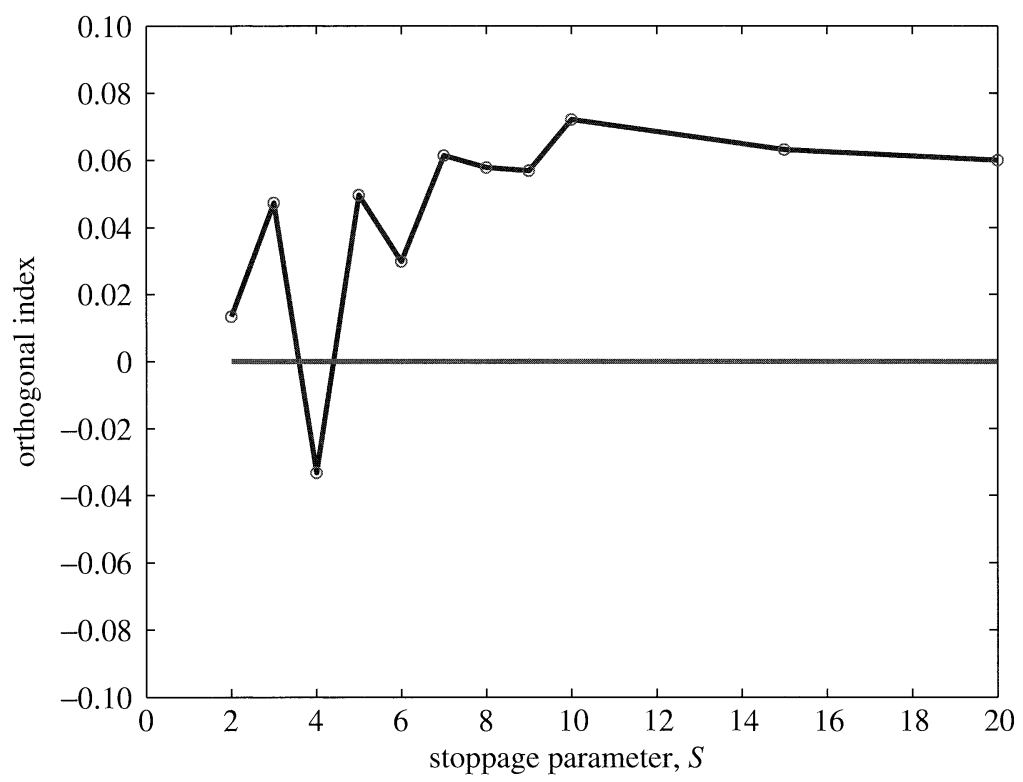

Figure 9. The OI variations as a function of the stoppage number for CEI cases.

the low-frequency range can never be recovered, a consequence again governed by the uncertainty principle.

Next, the same data will be processed by the EMD/HSA method. As recommended, the data are first passed through the sifting processes without invoking the intermittence test. Using extrema-based sifting with nine different combinations of sifting criteria, results were obtained with the variation of the OIs summarized in figure 3. By examining the OI as a function of $S$, it is evident that OI is not a function of $M$ as long as $M$ is larger than a critical number for each $S$. Or, more simply put, if a large enough $S$ is used, then the resulting IMF sets depend only on $S$. Here the result of the OI is presented as a function of $M$ and $S$. It can be seen that the OI for $S=1$ is uniformly poor at -0.253 . A different view of these results is presented as a series of cuts at specific $M$ values. The results are presented in figure 4 , with the values of each cut offset by 0.01 for easier visualization. For the $S$ numbers up to 10 , there is no difference between $M$ equal to 100 or 1000 . If $M$ is now decreased to 50 , however, the process cannot be fully completed when $S$ is larger than six. For $M$ less than 40, the $S$ number cannot be larger than three. For an optimal $S$ number, $M$ is set to 100 for the subsequent discussion. This selection is equivalent to setting no upper limit for the maximum number of siftings, and then letting the stopping criteria solely determine the final results. A total of $11 S$ numbers are selected: 2,3 , $4,5,6,7,8,9,10,15$ and 20 .

To examine these results in detail, the IMFs from the two extrema cases, CE $(100,2)$ and $\mathrm{CE}(100,10)$, are given in figure $5 a, b$. As can be seen, they do not have the same number of components, which makes averaging the IMF components impossible. In each IMF set, the main underlying mechanisms in the data are clear. The oscillations are dominated by the tides of both lunar and solar origin: $c_{1}$ denotes the semi-monthly tides; $c_{2}$, the monthly tides; $c_{3}$, the quasi-bimonthly tides; $c_{4}$, the semi-annual cycle; $c_{5}$, the annual cycle; $c_{6}$, the quasi-biennial cycle, and so on. But 
by examining the result more carefully, the mode mixing can be easily seen, i.e. there are signals of the quasi-bimonthly cycle mixed into the annual cycle, and there are signals of the annual mixed into the quasi-biennial cycle. These mode mixings will not improve by more sifting, as shown in the comparison between $\mathrm{CE}(100,2)$ and $\mathrm{CE}(100,10)$. Actually, more mode mixing occurs with more siftings, as shown in $\mathrm{CE}(100,10)$. As a result, the component representing the annual cycle is no longer continuous. The annual-cycle signal is distributed in as many as three IMF components $\left(c_{5}, c_{6}\right.$ and $\left.c_{7}\right)$. Furthermore, the semi-monthly tidal component in $\operatorname{CE}(100,10)$ is not continuous in the range from 1995 to 2001, which is the main cause of the subsequent mode mixing. Mode mixing was discussed in H98, and it was accepted as a consequence of sifting. The justification offered then was that once the IMFs are transformed into the Hilbert spectrum, the energy would find its proper place in the time-frequency plane. Yet, as also reported in H99, the mode mixing will introduce some fictitious variations in amplitude, and hence cause aliases in the instantaneous frequency, especially in the transitional region.

The most serious shortcoming for the uncontrolled sifting is that the number of IMF components obtained might be different with different choices of stopping criteria. Coupled with the mixed modes in any given IMF, this will cause the mean of the IMFs to be meaningless. This difficulty can be overcome partly by forcing the Hilbert spectra to have the same number of bins in time-frequency space and effect a mean in the Hilbert spectrum, even with different numbers in the final IMF sets from sifting criteria as in the CE series.

After averaging the Hilbert spectra, the individual case $\mathrm{CE}(100,2)$, the mean and the standard deviation of the Hilbert spectra are given in figure 6. From casual examination, there is very little difference between them. The individual spectrum and the mean are similar, and even the standard deviation spectrum is very similar to the mean. In order to show the difference between the mean and the standard deviation, their marginal spectra are shown in figure 7 . As can easily be seen, their magnitudes are drastically different. The similarity in the time-frequency presentation only indicates that the deviations occur where the energy is also concentrated.

Next, the marginal spectra of all 11 cases are plotted, together with the mean and two standard deviations about the mean offset by two decades lower for clarity, in figure 8. From the marginal Hilbert spectra, all the pertinent features of the underlying mechanisms can be identified: the monthly and annual cycles of the Earth-MoonSun interactions. The standard deviation ranges are reasonably low; therefore, there is a high degree of confidence in any of the 11 different trials.

To eliminate the mode mixing and examine the effects of using the intermittence test, sifting with the intermittence criterion is now invoked. For this example, the previous 11 different combinations of stopping criteria and the uniform intermittence criteria are selected as $\left(4,-1^{3}, 45^{2},-10\right)$. The first value, ' 4 ', means that half-cycles of four days are used as a criterion to filter any short-period phenomena produced by storms, for example. This four-day half-cycle is safely separated from the half-monthly tide. The next, $-1^{3}$, means that, for the next three components, no intermittence criterion is invoked. Thus, the sifting will simply follow the extrema produced by the half-monthly, monthly and bi-monthly tidal components, which are all very strong signals. The ' $45^{2}$ ' means that the intermittence test criterion of 45 data values was applied twice to remove any oscillation with a half-wave period less than 45 days, so that the semi-annual, annual and longer-

Proc. R. Soc. Lond. A (2003) 
(a)

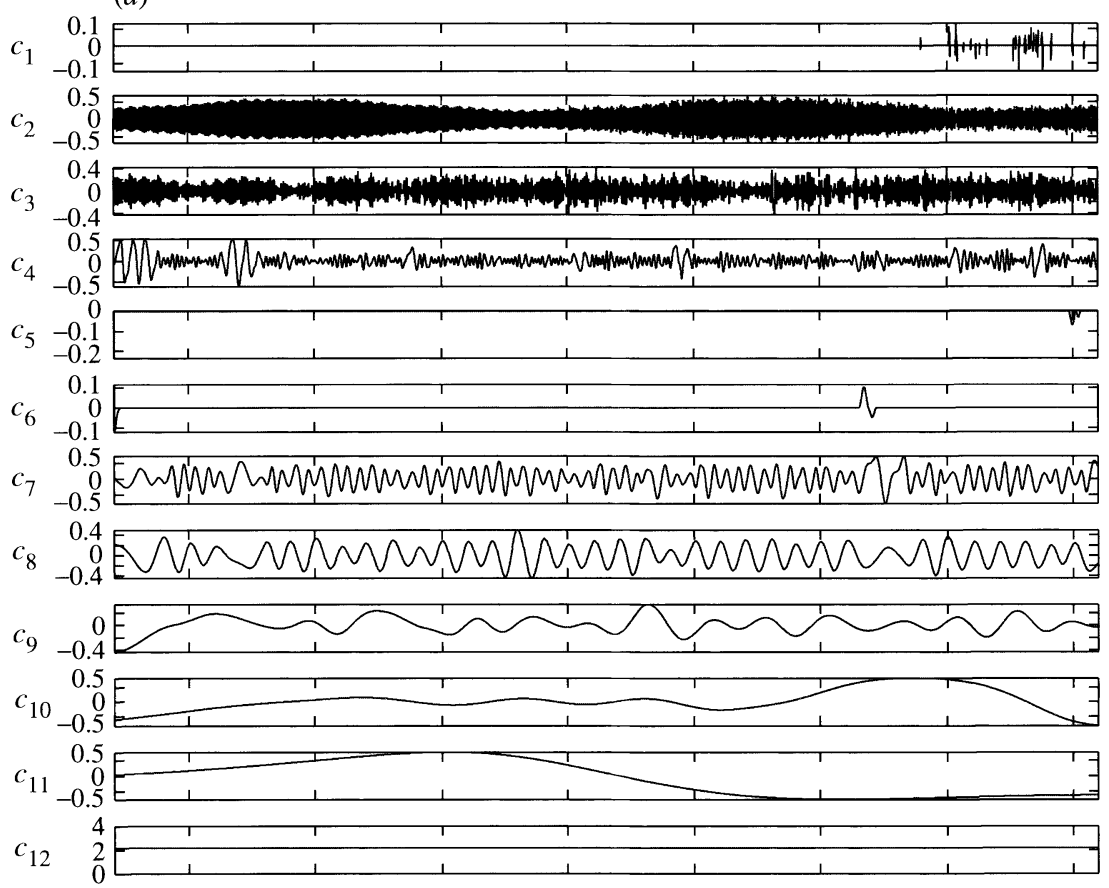

(b)

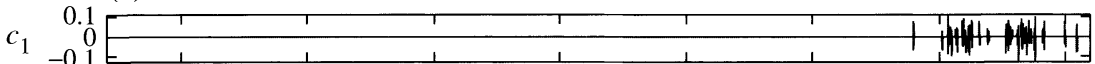

$c_{2} \begin{array}{r}0.5 \\ 0.5\end{array}$

$c_{3} \underset{0}{0.4}{ }_{0}^{0}$

$c_{4}{ }_{-0.2}^{0.2}$ End (n)

$c_{5} \begin{array}{r}0.2 \\ \hline-1\end{array}$

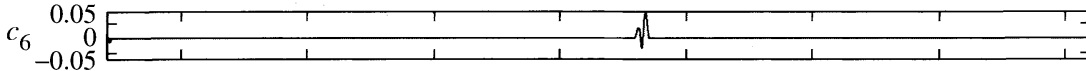

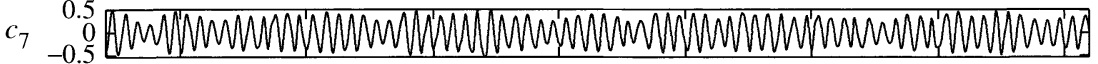

$c_{0.5}^{0.5}$
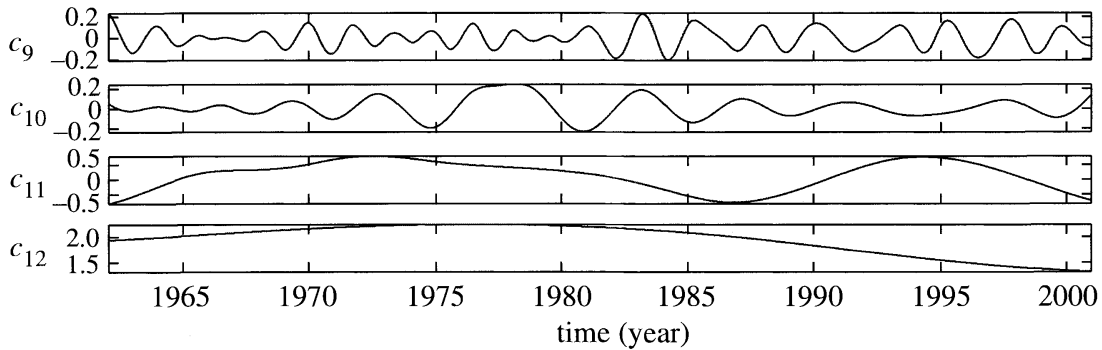

Figure 10. The IMF components from extrema-based sifting with intermittence test. (a) An individual case: $\operatorname{CEI}\left(100,2 ; 4,-1^{3}, 45^{3},-10\right) ;(b)$ an individual case: $\operatorname{CEI}\left(100,10 ; 4,-1^{3}, 45^{3},-10\right)$. 

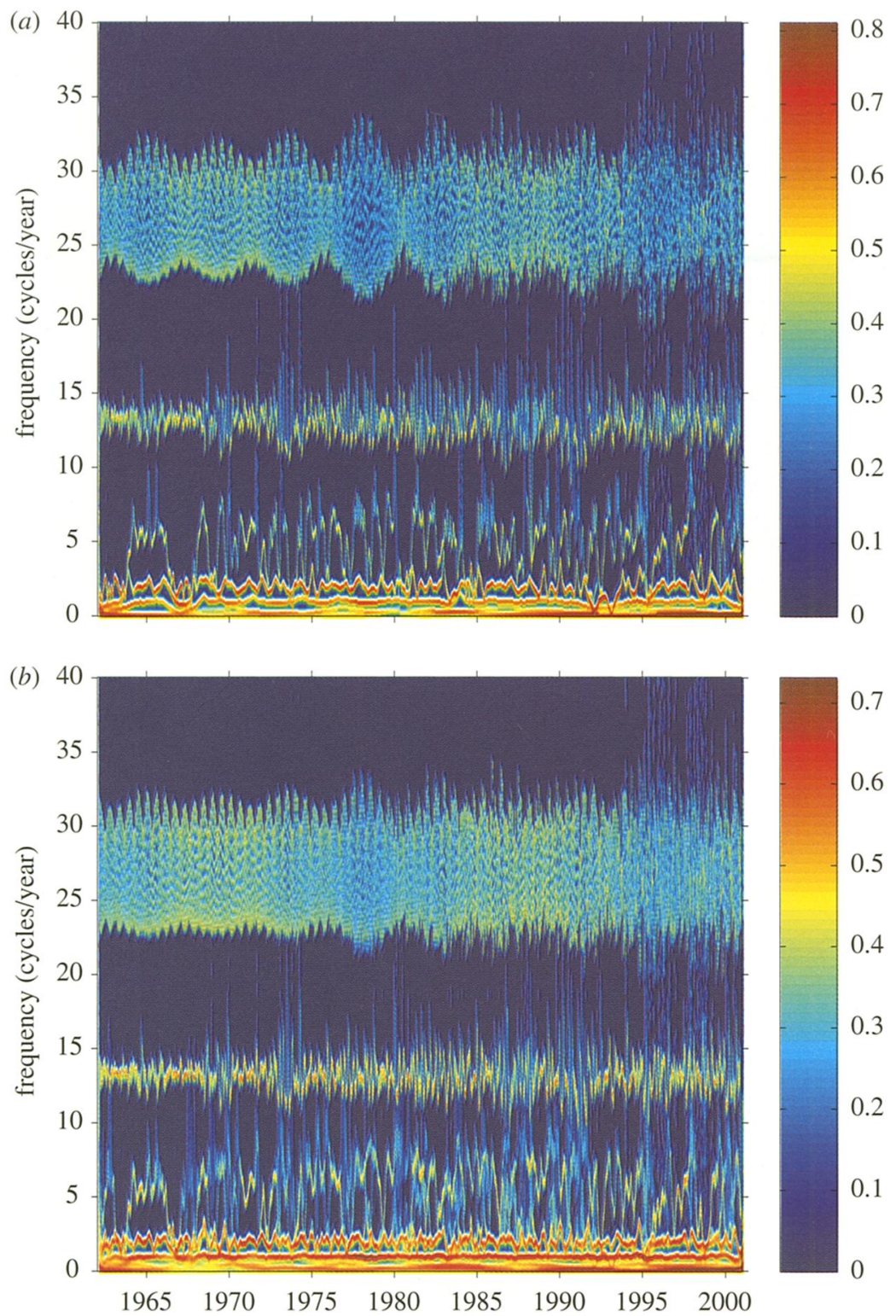

Figure 11. The Hilbert spectra constructed from the CEI series of siftings with $7 \times 7$ smoothing. (a) An individual case for the Hilbert spectrum based on CEI $\left(100,2 ; 4,-1^{3}, 45^{3},-10\right)$; $(b)$ the mean Hilbert spectra of 11 different siftings based on the CEI series.

period cycles would not be contaminated. Finally, the intermittence criterion was stopped for the rest of the IMF components (indicated by ' -10 ', which here has no other meaning beyond a recognized decision-switch value in the processing). The OIs for all the cases are shown in figure 9. Using OI alone, it cannot be determined which case is better. These properties will need study through other means. 


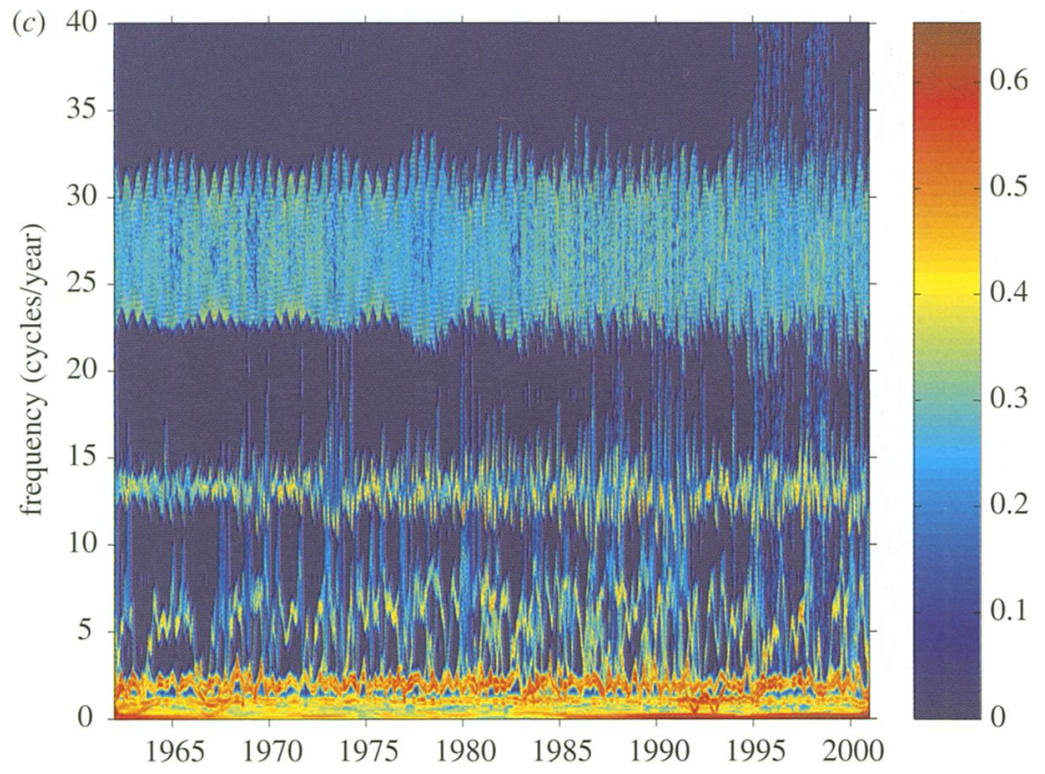

Figure 11. (Cont.) (c) the standard deviation of the Hilbert spectra from 11 different siftings based on the CEI series.

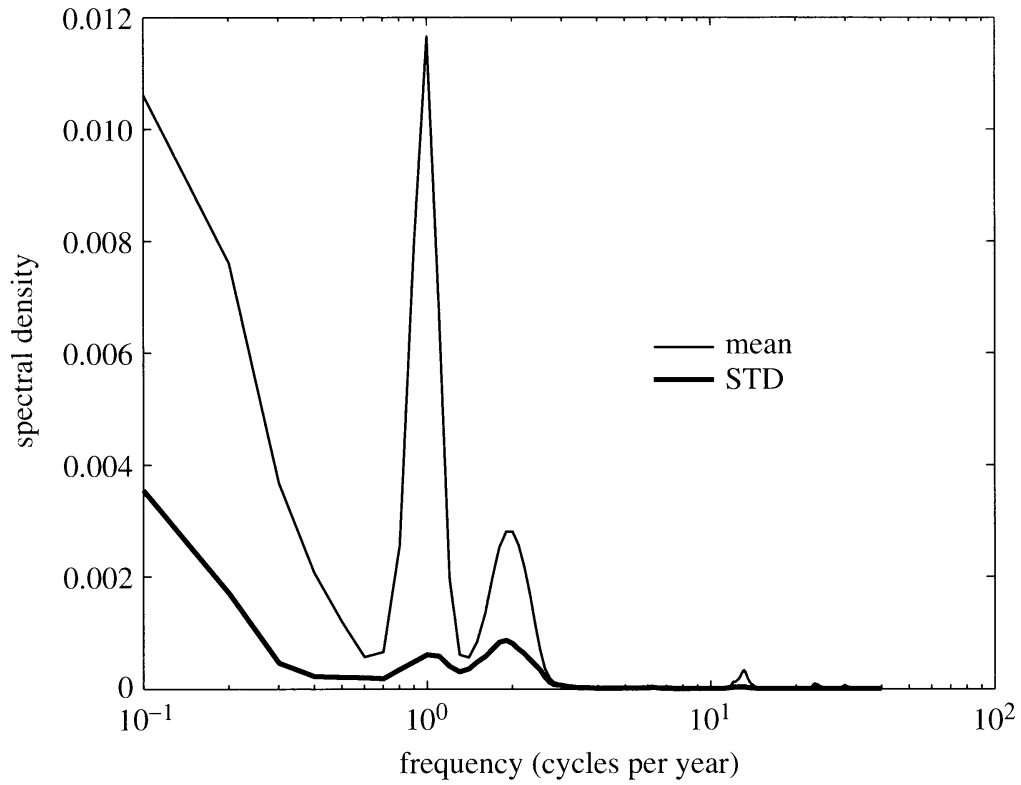

Figure 12. The marginal spectra for the CEI series from the mean Hilbert spectrum and the time-frequency representation of the standard deviation of the Hilbert spectra.

The IMFs in two extrema cases are next examined for the effects of sifting with the intermittence test, $\mathrm{CEI}\left(100,2 ; 4,-1^{3}, 45^{3},-10\right)$ and $\mathrm{CEI}\left(100,10 ; 4,-1^{3}, 45^{3},-10\right)$ as given in figure $10 a, b$, respectively. With the intermittence test, all siftings produce twelve IMFs even with different sifting criteria. As can be seen, the main underlying 
mechanisms in the data are much clearer than in the corresponding CE series: $c_{1}$ represents all the variations with periods of less than eight days. The logical explanation is the perturbation of the Earth's rotation introduced by the perturbation of shortperiod storms, as studied by Clark et al. (1998). Then $c_{2}$ denotes the semi-monthly tides; $c_{3}$, the monthly tides; $c_{4}$, the quasi-bimonthly tides; $c_{7}$, the semi-annual cycle; $c_{8}$, the annual cycle; $c_{9}$, the quasi-biennial cycle, and so forth. There are two components, $c_{5}$ and $c_{6}$, of unknown causes, at present representing intermittent oscillations with periods less than 90 days. Their generating mechanisms need to be explored in the future but, to ensure the continuity of the known semi-annual and annual components, they have to be eliminated at present. The difference between the CEI and $\mathrm{CE}$ cases is also clear: there are almost no mode mixings in the semi-monthly, the monthly, the semi-annual, the annual and the quasi-biennial cycle components, except in the $\operatorname{CEI}\left(100,2 ; 4,-1^{3}, 45^{3},-10\right)$ case. Otherwise, the results of even the two extrema cases look almost identical. All IMF sets have the same numbers of components up to $S=15$. Even when the IMF component number increases by one for $S=20$, the average and the standard deviation of the IMFs for the components up to $c_{9}$ can still be computed. Furthermore, the mean for the Hilbert spectrum for the CEI series can be obtained.

The Hilbert spectrum of an individual case, the mean and the standard deviation are given in figure 11. Again, it is hard to distinguish either the individual case from the mean, or the mean from the standard deviations. Surprisingly, the difference between them and even among the CEI and CE series is not that obvious. Only through careful scrutiny can the more subtle variations be detected: first, the energy distribution for the quasi-bimonthly tide is much more diffuse. Second, and even more significantly, the annual and the quasi-biennial cycles are continuous and smooth in the CEI case in figure 11, but fragmented for the CE series in figure 6 . This is the consequence of the mode mixing. While the Hilbert spectral representation has indeed alleviated the difficulties caused by the mode mixing through assigning energy to its proper time-frequency location according to its instantaneous frequency value as discussed by H98, there are unavoidable aliases caused by switching back and forth in the time-frequency space induced by the mode mixing. Such a frequency alias near the switching points can be seen most clearly in the period covering 1990 2000. On the other hand, the nearly continuous annual cycles in the CEI series speak powerfully for the sifting with the intermittency test: if the existence of a specific frequency is known a priori or suggested by extensive mode mixing, the intermittence test should be invoked.

When considering the results in time-frequency presentations as in figure 11, it can be noted that the standard deviation values are in general very small, except for the first three IMF components in the period covering 1990-2000. This almost suggests that the data quality is changing for that period, as will be discussed presently. The higher standard deviation suggests that more-detailed data might have been used in the construction of the LOD dataset. The marginal spectra for both the mean and the standard deviation are given in figure 12. Compared with figure 7 , the difference can be seen between the mean and the standard deviation for the case with intermittence more pronounced. The individual and the mean marginal spectrum are given in figure 13. Again, the dynamical range of the marginal spectrum is improved for the cases with intermittence over the cases without the intermittence test. Comparisons can also be made between the marginal Hilbert and Fourier spectra. As discussed 
in H98 and H99, the difference is clear: while the Fourier spectrum gives sharply defined peaks, they represent only the mean frequency. The Fourier spectrum gives no indications of the temporal variations of the amplitudes at the particular frequency band in the natural processes. All deviations from the few mean-frequency bands are represented by the harmonics and the broad-base energy distribution. When the confidence limit is computed, the data must be subdivided into short subsections. As a result, longer-period phenomena cannot be resolved. The marginal spectra of the Hilbert spectrum with confidence limits, however, use the full-length data; therefore, they still retain all the resolution power over the range of all the frequencies.

As the CE-series have different numbers of IMFs, averaging of the IMFs component by component is thus impossible. But for the CEI cases, the average of the IMFs can be obtained. A more detailed comparison of the IMFs is given in figures 14 and 15 . In figure 14, the mean and the standard deviation of each IMF component are given. Obviously, for the annual component, $c_{8}$, the standard deviation has two visible local maximum ranges over 1965-1970 and 1990-1995, other than the end zone. In figure 15, the individual and mean envelopes of the annual-cycle component are plotted for all 11 CEI cases. The mean clearly shows a fluctuation. Interestingly, each of these envelope peaks corresponds to a recognized El Niño event. This should not be a total surprise, for the effect of tidal and El Niño events on the rotation speed of the Earth has been reported previously by Chao (1989) and Clark et al. (1998). What is new about this approach is that the variations come out here as a result of the sifting process. Furthermore, the more intriguing feature of the sifting process used is that the larger-than-average standard deviations occur over the ranges 1965-1970 and 1990-1995 in figure 15. Both of these periods coincide with anomalies of the El Niño sequence: the equatorial waters show continuous weak warming events according to the El Niño summary by the National Oceanic and Atmospheric Administration of the US Government. Therefore, the sifting process identifies not only the El Niño events, but also the anomalies: a surprising result testifying to the power of this new approach.

It is interesting to note that the magnitudes of the LOD variation are not in direct proportion to the strength of the El Niño events. This might be caused by the fact that the influence of the El Niño events on the rotational speed is primarily from the angular momentum changes induced by the variations of the atmospheric system (Chao 1989), while the strength of the El Niño events is traditionally measured by the sea-surface temperature anomalies (Philander 1990). Therefore, this result only tentatively implies that the oceanic signature of El Niño is only weakly and indirectly coupled with the atmospheric dynamics. This could be used as an indication that the oceanic El Niño events are primarily oceanic events, and the atmospheric dynamics features are consequences of the oceanic changes coupled nonlinearly and disproportionately. The coupling, nevertheless, is strong enough to trigger such atmospheric disturbances as to change the rotational speed of the Earth. As the causal relationship between oceanic and atmospheric dynamics in the El Niño event is a critical but unsettled issue, it needs to be explored further in light of the present results.

The LOD data have been used to illustrate the applications of the EMD/HSA (or HHT) method. Newly added to the analysis was a statistical measure of the confidence limit. This study shows that, when the EMD is applied with various sifting criteria, the same dataset can be used to produce a statistical mean and confidence limits without invoking the ergodic assumption. All that is remaining is to prove that 


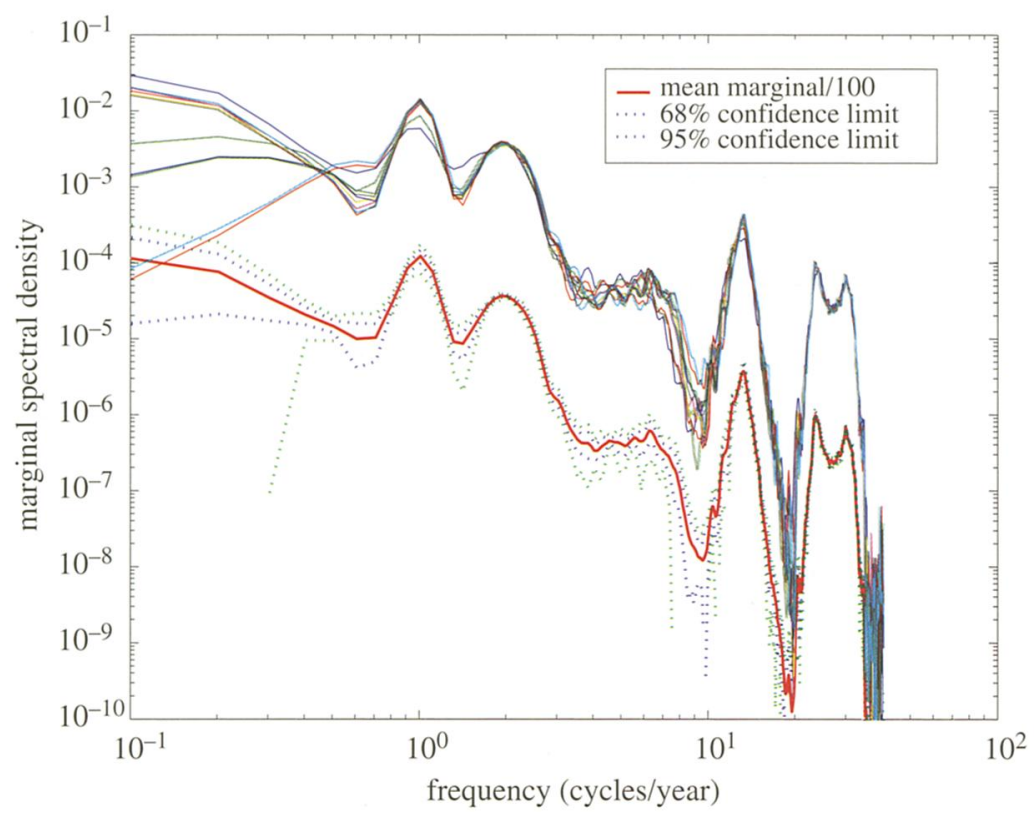

Figure 13. All 11 marginal Hilbert spectra for the CEI series, and their mean (shifted down two decades) with the two-standard-deviations confidence limit.

the sampling strategy used is a fair one. In the next section, the sampling strategy will be examined and a range of stopping criteria that will give the optimal sifting criterion will be derived from it.

\section{The sampling strategy and the optimal stopping criteria}

In the above discussions, the ensemble mean used to derive the confidence limit was based solely on the selection of different stopping criteria. From the OI indices check, it can be seen that each selection of the stopping criterion indeed produces a distinct IMF set. In order to have high confidence in the result, the ensemble of samplings must be shown to cover all valid variations. As there are infinitely many selections of stopping criteria, the range of choices must be limited. A limitation can be established by considering the effects of the sifting. Based on the discussions here, there are two beneficial effects: to eliminate the riding waves, and to force symmetry. But there is also the side effect of smoothing the amplitudes of the neighbouring oscillations. Indeed, through examination of the individual envelopes of the annual-cycle IMFs, the envelope for the $\operatorname{CEI}(100,20)$ case was found to be much smoother than that for the CEI $(100,2)$ case. The smoothness effects appear in two ways. Firstly, the amplitude variation is smaller, and, secondly, the absolute values of the peaks are also more even. Both of these trends tend to obliterate the natural perturbations caused by El Niño. Therefore, even if the OIs of all the cases are equally acceptable, a range of valid IMFs has been established when the stopping criterion approaches 20 .

An examination of the variations of the deviation of the individual cases from the mean will be considered next, to sec if there is a range of stopping criteria that produces relatively stable results in either the IMFs or the Hilbert spectrum. This 
(a)

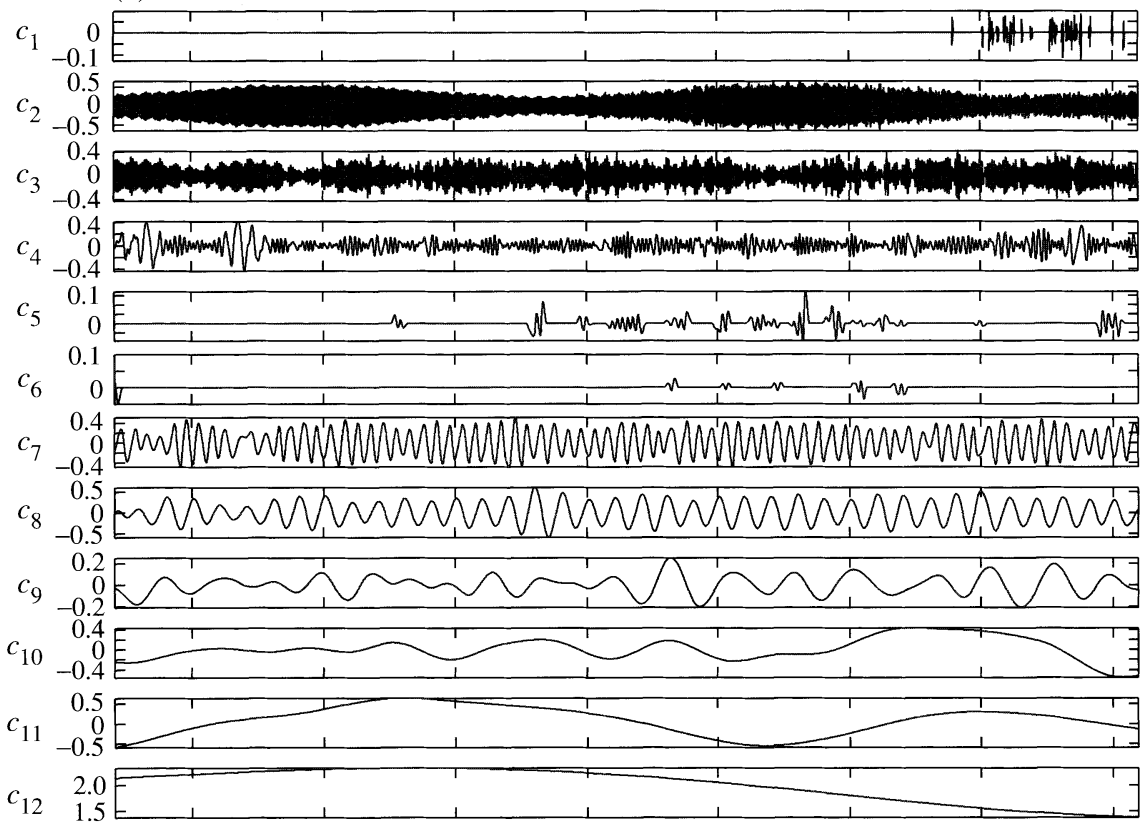

(b)

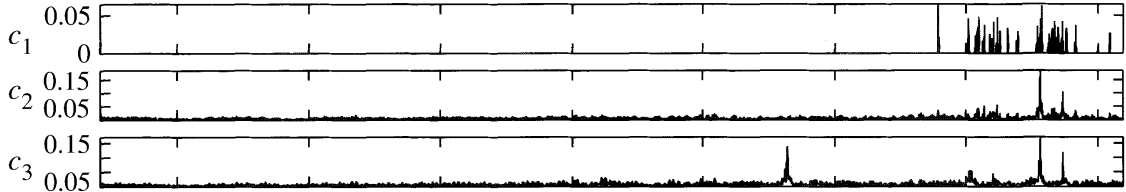

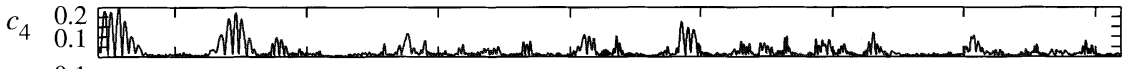

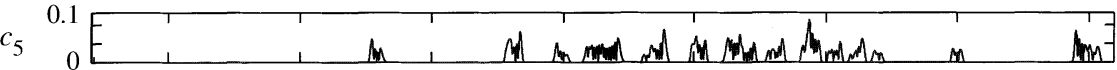

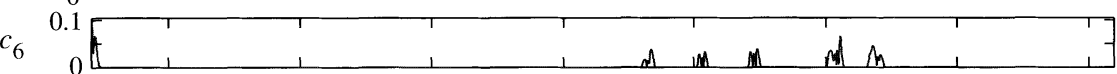

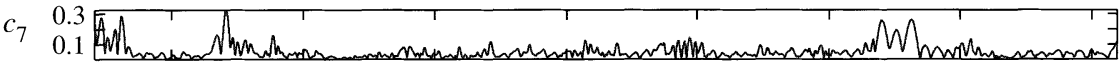

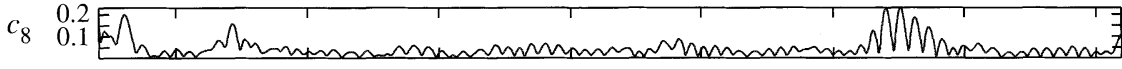

$c_{9} \quad \begin{aligned} & 0.2 \\ & 0.1\end{aligned}$

$c_{10} \begin{array}{ll}0.4 \\ 0.2\end{array}$

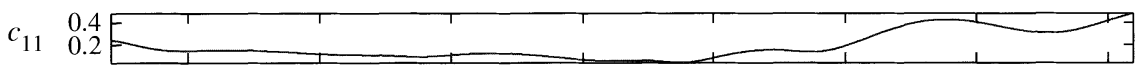

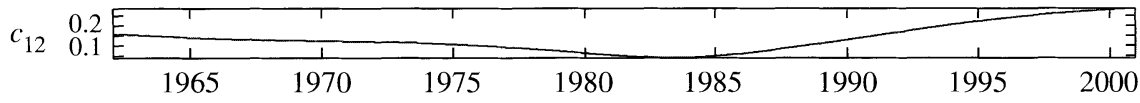
time (year)

Figure 14. The mean and standard deviation of the IMFs from all 11 cases based on the CEI series. (a) The mean IMF; $(b)$ the standard deviation. Notice the large values in $c_{8}$ at the mid 1960 s and early 1990s. 
can be accomplished by first computing the mean from the samples of any quantity, $V(S)$, as a function of the stopping criterion, $S$ :

$$
\langle V\rangle=\frac{1}{N} \sum_{j=1}^{N} V\left(S_{j}\right) .
$$

The squared deviation of each individual case can be computed from this mean by using

$$
\operatorname{sd}\left(S_{j}\right)=\left[V\left(S_{j}\right)-\langle V\rangle\right]^{2} .
$$

Figure $16 a$ shows the $\operatorname{sd}(S)$ values for the annual cycle. There is a clear range of minimal $\operatorname{sd}(S)$ values covering the $S$ choices from 4 to 10 . Over this range, the IMFs are relatively stable. Beyond this range, the $\operatorname{sd}(S)$ values are seen to increase. This increase of $\operatorname{sd}(S)$ values for higher $S$ values also coincides with smoothness of the envelope obtained in the processing. For the lower $S$ values, a failure to sift out a continuous annual cycle is seen in the processing. Figure $16 b$ presents the $\operatorname{sd}(S)$ values for the semi-monthly tidal cycle. There is again a clear range of minimal $\operatorname{sd}(S)$, covering the $S$ values from 5 to 10 . Over this range, the IMFs are relatively stable with similar increasing trends at both ends. Such trends suggest that the range 510 is a good choice for the stopping criteria, where the result will have a minimal difference from the computed mean.

The deviation squared, $\operatorname{sd}(S)$, variation for the Hilbert spectra will be examined next. Figure $16 c$ plots the $\operatorname{sd}(S)$ values for the CEI cases. Here a relatively flat region of $\operatorname{sd}(S)$ can be seen for $S$ choices from 3 to 8 . The corresponding result for the CE cases is given in figure $16 \mathrm{~d}$. Although there is an extended range of relatively low $\operatorname{sd}(S)$ values, the range $4-8$ is still a good choice for stable minimal $\operatorname{sd}(S)$ values.

The deviation squared value, $\operatorname{sd}(S)$, for the annual and semi-monthly tidal cycle IMFs, and the Hilbert spectra for the CEI and CE cases are plotted in figure 16 .

Up to now, only the case with known fixed cycles has been examined. To test the approach for general cases, an earthquake data record was examined. The data are taken from Station TCU129, located near Chi-Chi, Taiwan. The earthquake event occurred on 21 September 1999. This case has been studied in some detail by Huang et al. (2001). Here only the east-west component is used to illustrate the effect of the stopping criteria. This is a very severe earthquake, for the maximum acceleration reaches almost $1 \mathrm{~g}$, as indicated in the data given in figure 17 . As there is no fixed time-scale, the intermittence test is irrelevant. Sifting without an intermittence test results in the $\operatorname{sd}(S)$ values as shown in figure 18. A minimal $\operatorname{sd}(S)$ range $2-10$ is easily seen.

\section{Discussion}

The results presented imply that the selection of $S$ used is uniform and covers the full valid range of possible choices. Therefore, the mean obtained is valid, from the point of view of a simple random sample. Because the mean is an ensemble one, the averaging process is simply the sum of the random variables multiplied by its individual probability density function. Each case is treated as equal; consequently, the mean is a simple arithmetic average, an estimate. The condition for validity for this case is very much different from that under the ergodic assumption. According 
to Halmos (1956), the ergodic assumption can only be true when the data are from a linear and stationary process, and the data have to be decomposable (that is divided into $n$-independent subsections). However, the full data stream can be used here and the processing reveals all the possible scales the original data represent, because the ergodicity was not invoked.

A statistical measure in the form of a confidence limit has been defined based on the IMFs generated by different stopping criteria. This result is interesting in itself, for the confidence limit has been derived without invoking the ergodic assumption. Rather, various sifting criteria have been used to obtain an ensemble of IMF sets, all from the same data. Furthermore, the confidence limit is still a function of time and frequency. The confidence limit can be applied to individual IMF components, if the number of IMF components in the ensemble is the same. The confidence limit can be also applied to Hilbert spectra whenever the number of the IMF components is not the same in different IMF sets. In the examples presented here, the stopping criteria chosen for the sifting are in the general range 4-8. This is reasonably close to 5 , the value that has been used the most.

It should be pointed out that the confidence limit as discussed here is specific to the methodology used. This is true for the Fourier spectral methods in use as well. Other forms of a confidence limit can also be defined. If, instead of a stopping criterion, a different spline was used in the sifting operation, then another ensemble of samples may be obtained. How such a confidence limit would vary from the present one is a totally different problem. The spline fitting has been extensively discussed in H98 and H99. Experience has indicated that the natural spline is the most reasonable one to select; therefore, the present confidence limit as presented here is offered as both reasonable and the one to adopt.

Finally, an added advantage of the EMD/HSA method should be considered. Due to its superior time-frequency resolution, the method can reveal the changing characteristics of the data in two aspects. The first is on the data quality. As has been seen in the Hilbert spectral representation of the LOD data, the energy distribution pattern shows very different characteristics over the time-span: the distribution is very diffuse over the period covering 1990-2000, indicating that the data quality might have undergone certain changes. Furthermore, if the envelope of the $c_{1}$ IMF component from the mean CEI series in figure 12 is examined, the envelope characteristics are seen to change, starting around 1985, and the variations become even more prominent after 1995. Do these changes in the data represent part of the underlying physical changes? It is impossible to say, for the underlying mechanism is only the interaction of the Earth, Moon and Sun with minor perturbations of the atmospheric processes. Therefore, the change here should most logically be attributed to a data quality change. It could be the result of including more-detailed measurements, new data sources, or more model results, for example. No other data-analysis methods can reveal these changes as readily as the EMD/HSA method did in this study. This is clearly another advantage of the EMD/HSA approach. The second aspect to consider concerns the natural processes. It is totally unexpected to find that the large standard deviation on the annual cycle of the IMF from the CEI cases coincide with the uncertainty of the El Niño events over the mid 1960s and early 1990s. The results therefore show that the EMD/HSA method with various stopping criteria should be explored further to see if more information can indeed be extracted from a given dataset. 


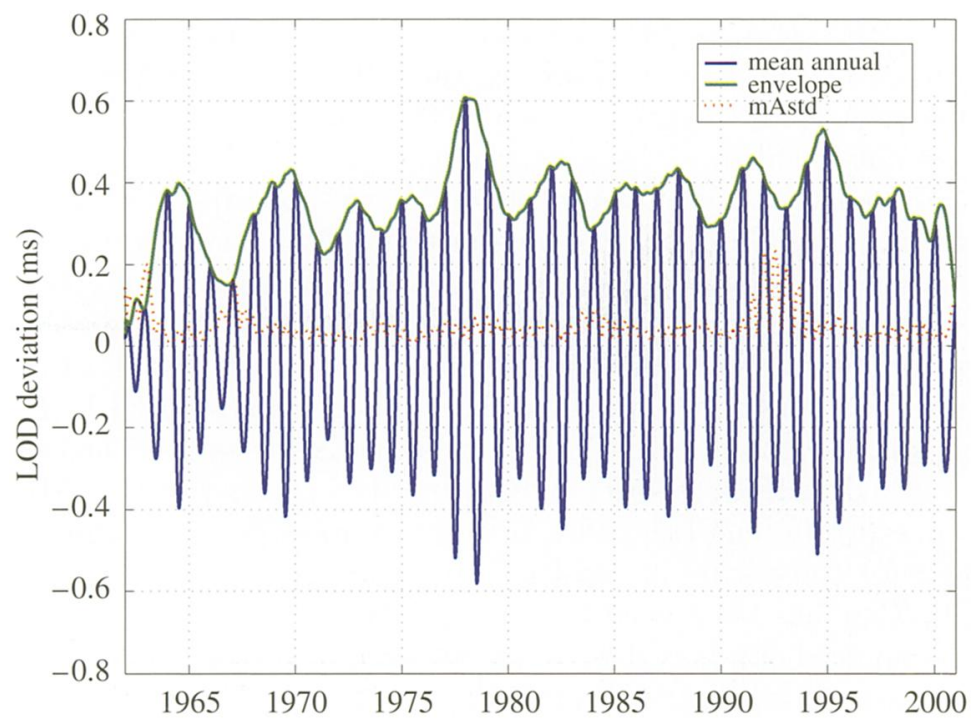

Figure 15. The mean annual-cycle IMF from the CEI series and its envelope and the standard deviation. Notice that each peak of the envelope corresponds to an El Niño event, and the high values of the standard deviation during the periods of the mid 1960s and early 1990s.
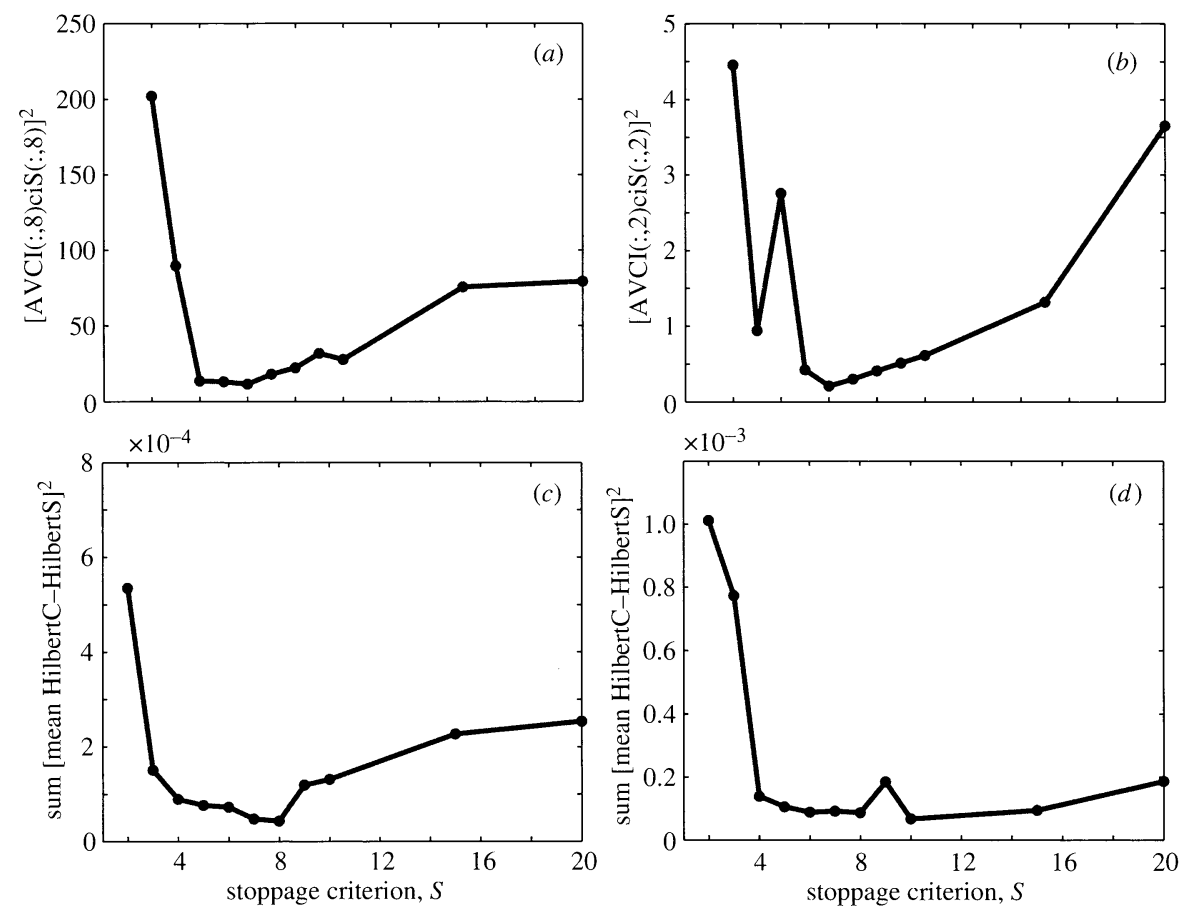

Figure 16. The squared deviation of the individual cases from the ensemble mean for the following quantities as functions of stoppage number: (a) IMF annual cycle, CEI series; $(b)$ IMF semi-monthly cycle, CEI series; $(c)$ Hilbert spectra, CEI series; $(d)$ Hilbert spectra, CE series. 


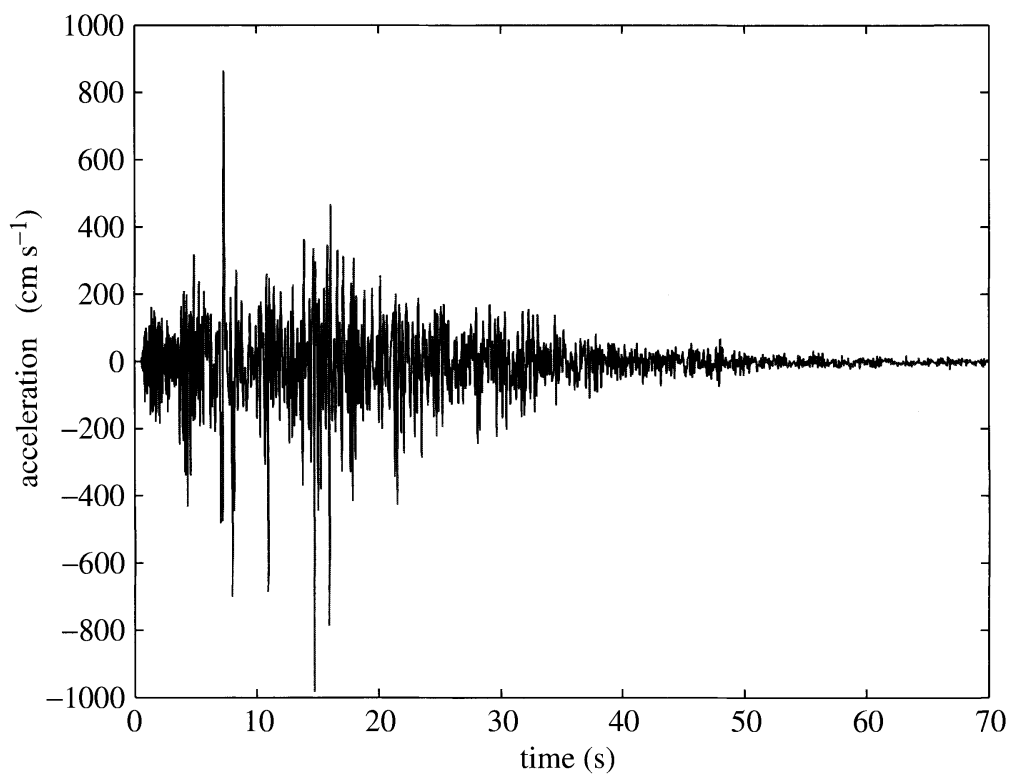

Figure 17. The east-west acceleration record of the earthquake event at station TCU129, Chi-Chi, Taiwan, 21 September 1999.

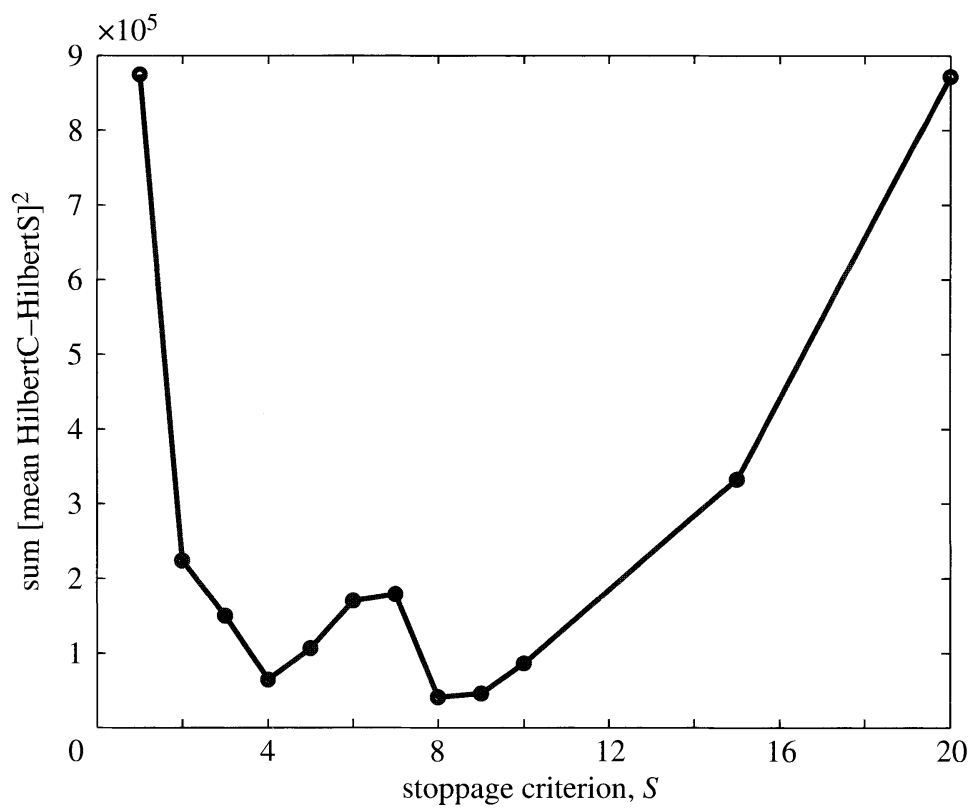

Figure 18. The squared deviation of the individual cases from the ensemble mean for 11 Hilbert spectra from the earthquake record given in figure 17.

\section{Conclusions}

Sifting is a general method of decomposing a given dataset into underlying scales of various sizes. By varying the chosen parameters in the sifting process, infinitely many 
IMF sets can be generated, or at least as many as needed. In this paper, these features of the EMD/HSA method have been used in a constructive way to examine data by introducing a statistical measure of the confidence limit from a single set of nonstationary and nonlinear data without invoking the ergodic assumption. With the help of the newly introduced confidence limit, a stable range of stopping criteria for the first step of the EMD/HSA method (the EMD-sifting operation) has also been established. This statistical measure has helped to make the EMD/HSA method more definitive. The scale parameters for the intermittence test are phenomenon dependent. Typically, a decomposition of the data (the first step, EMD) should be first made without the intermittence test. If mode mixing is clearly seen to occur, the scales should be determined from that result, and the selection made based on the time-scale for the intermittence test, so that each IMF can contain results of one narrow time-scale range. In the case of LOD, the decision is an easy one, for there are definite cycles. For other phenomena, it might not be so apparent. An indication of the scales present can also be determined from the marginal spectrum obtained through the EMD/HSA method applied without intermittence by identifying the peaks in that spectrum as an indication of the existence of relatively narrow band periodic variations. The scale parameter can be determined accordingly.

With these additions and improvements, we have increased the rigour of the EMD/HSA method, and thus also made it more robust and useful.

This research is performed under the support by the NASA RTOP Program through the Oceanic Processes Program Office. N.E.H. and S.R.L. express their sincere appreciation for the consistent support from Dr E. Lindstrom of NASA Headquarters. N.E.H. is also supported in part by an ONR Grant (no. N00014-98-F-0412) from the Processes and Prediction Program, and in part by a NOAA grant. S.S.P.S. is supported by a Senior NRC Fellowship at the Laboratory for Atmospheres, NASA Goddard Space Flight Center, while on leave from the University of Alberta, and in part by a NOAA grant. W.Q. is supported in part through a grant to California Institute of Technology from NASA. K.L.F. expresses thanks for support by a grant from the National Research Council, Taiwan, ROC. We would like to also thank Dr Ben Chao for his many encouragements, and Dr Richard Gross for generously providing the LOD data, without which the present study would not be possible. Additional thanks go to Abigail Long for editorial help in the final preparations of the manuscript.

\section{References}

Chao, F. 1989 Length-of-day variations caused by El Niño-southern oscillation and quasi-biennial oscillation. Science 243, 923-925.

Clark, T. A., Ma, C., Ryan, J. W., Chao, B. F., Gipson, J. M., MacMillan, D. S., Vandenberg, N. R., Eubanks, T. M. \& Niell, A. E. 1998 Earth rotation measurement yields valuable information about the dynamics of the Earth system. Eos 79, 205-209.

Frisch, U. 1995 Turbulence. Cambridge University Press.

Gloersen, P. \& Huang, N. E. 1999 In search of an elusive Antarctic circumpolar wave in sea ice extents: 1978-1996. Polar Res. 18, 167-173.

Gross, R. S. 1996 Combinations of Earth orientation measurements: SPACE94, COMB94, and POLE94. J. Geophys. Res. 101, 8729-8740.

Gross, R. S. 2000 Combinations of Earth orientation measurements: SPACE97, COMB97, and POLE97. J. Geodesy 73, 627-637.

Gross, R. S. 2001 Combinations of Earth orientation measurements: SPACE2000, COMB2000, and POLE2000. JPL Publication 01-2. Jet Propulsion Laboratory, Pasadena, CA. 
Gross, R. S., Eubanks, T. M., Steppe, J. A., Freedman, A. P., Dickey, J. O. \& Runge, T. F. 1998 A Kalman filter-based approach to combining independent Earth orientation series. $J$. Geodesy 72, 215-235.

Halmos, P. R. 1956 Lectures on ergodic theory. Tokyo: The Mathematical Society of Japan.

Hu, C. C., Miau, J. J. \& Chou, J. H. 2002 Instantaneous vortex-shedding behaviour in periodically varying flow. Proc. R. Soc. Lond. A 458, 911-932.

Huang, N. E. 2001 Applications of Hilbert-Huang Transform for speech analysis, synthesis, music signal enhancement, and machine health monitoring. US patent. (Submitted.)

Huang, N. E., Long, S. R. \& Shen, Z. 1996 Frequency downshift in nonlinear water wave evolution. Adv. Appl. Mech. 32, 59-117.

$\rightarrow$ Huang, N. E., Shen, Z., Long, S. R., Wu, M. C., Shih, E. H., Zheng, Q., Tung, C. C. \& Liu, H. H. $1998 a$ The empirical mode decomposition method and the Hilbert spectrum for non-stationary time series analysis. Proc. R. Soc. Lond. A 454, 903-995.

Huang, W., Shen, Z., Huang, N. E. \& Fung, Y. C. $1998 b$ Use of intrinsic modes in biology: examples of indicial response of pulmonary blood pressure to \pm step hypoxia. Proc. Natl Acad. Sci. USA 95, 12 766-12 771.

Huang, N. E., Shen, Z. \& Long, S. R. 1999a A new view of nonlinear water waves: the Hilbert spectrum. A. Rev. Fluid Mech. 31, 417-457.

$\rightarrow$ Huang, W., Shen, Z., Huang, N. E. \& Fung, Y. C. $1999 b$ Nonlinear indicial response of complex nonstationary oscillations as pulmonary hypertension responding to step hypoxia. Proc. Natl Acad. Sci. USA 96, 1834-1839.

Huang, N. E., Shih, H. H., Shen, Z., Long, S. R. \& Fan, K. L. 2000 The ages of large-amplitude coastal seiches on the Caribbean coast of Puerto Rico. J. Phys. Oceanogr. 30, 2001-2012.

Huang, N. E., Chern, C. C., Huang, K., Salvino, L., Long, S. R. \& Fan, K. L. 2001 Spectral analysis of the Chi-Chi earthquake data: station TUC129, Taiwan, September 21, 1999. Bull. Seism. Soc. Am. 91, 1310-1338.

Kantha, L. H., Stewart, J. S. \& Desai, S. D. 1998 Long-period lunar fortnightly and monthly ocean tides. J. Geophys. Res. 103, 12 639-12647.

Loh, C. H., Wu, T. C. \& Huang, N. E. 2001 Application of EMD-HHT method to identify near-fault ground motion characteristics and structural responses. Bull. Seism. Soc. Am. 91, 1339-1357.

Philander, S. G. 1990 El Niño, La Niña, and the Southern Oscillation. International Geophysics Series, vol. 46. Academic Press.

Wu, M. L. C., Schubert, S. \& Huang, N. E. 1999 The development of the South Asian summer monsoon and the intraseasonal oscillation. J. Clim. 12, 2054-2075.

$\rightarrow$ Yoder, C. F., Williams, J. G. \& Parke, M. E. 1981 Tidal variations of Earth rotation. J. Geophys. Res. 86, 881-891.

As this paper exceeds the maximum length normally permitted, the authors have agreed to contribute to production costs. 